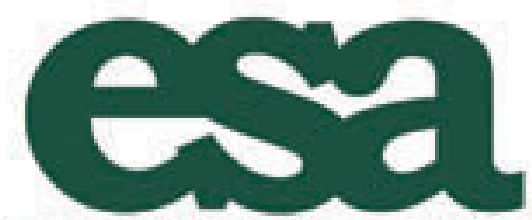

Promoting the Science of Ecology

Remote Sensing of Forest Biophysical Structure Using Mixture Decomposition and Geometric Reflectance Models

Author(s): Forrest G. Hall, Yosio E. Shimabukuro, Karl F. Huemmrich

Source: Ecological Applications, Vol. 5, No. 4 (Nov., 1995), pp. 993-1013

Published by: Ecological Society of America

Stable URL: http://www.jstor.org/stable/2269350

Accessed: $31 / 08 / 2011$ 10:38

Your use of the JSTOR archive indicates your acceptance of the Terms \& Conditions of Use, available at http://www.jstor.org/page/info/about/policies/terms.jsp

JSTOR is a not-for-profit service that helps scholars, researchers, and students discover, use, and build upon a wide range of content in a trusted digital archive. We use information technology and tools to increase productivity and facilitate new forms of scholarship. For more information about JSTOR, please contact support@jstor.org. 


\title{
REMOTE SENSING OF FOREST BIOPHYSICAL STRUCTURE USING MIXTURE DECOMPOSITION AND GEOMETRIC REFLECTANCE MODELS ${ }^{1}$
}

\author{
FORREST G. HALL \\ Goddard Space Flight Center, Greenbelt, Maryland 20771 USA \\ Yosio E. Shimabukuro \\ Instituto Nacional de Pesquisas Espaciais, Sao Jose dos Campos-SP Brasil \\ KARL F. HUEMMRICH \\ Hughes STX, Greenbelt, Maryland 20771 USA
}

\begin{abstract}
Using geometric shadow and linear mixture models we develop and evaluate an algorithm to infer several important structural parameters of stands of black spruce (Picea mariana), the most common boreal forest dominant. We show, first, that stand reflectances for this species can be represented as linear combinations of the reflectances of more elemental radiometric components: sunlit crowns, sunlit background, and shadow. Secondly, using a geometric model, we calculate how the fractions of these radiometric elements covary with each other. Then, using hand-held measurements of the reflectances of the sunlit background, sphagnum moss (Sphagnum spp.), and assuming shadow reflectance to be that of deep, clear lakes, we infer the reflectance of sunlit crowns from the geometric shadow model and lowaltitude reflectance measurements acquired by a helicopter-mounted radiometer. Next, we assume that the reflectance for all black spruce stands is simply a linear combination of shadow, sunlit crown, and sunlit background reflectance, weighted in proportion to the relative areal fractions of these pixel elements. We then solve a set of linear equations for the areal fractions of these elements using as input helicopter observations of total stand reflectance.

Using this algorithm, we infer the values for the areal proportions of sunlit canopy, sunlit background, and shadow for 31 black spruce stands of varying biomass density, net primary productivity, etc. We show empirically and theoretically that the areal proportions of these radiometric elements are related to a number of stand biophysical characteristics. Specifically, the shadow fraction is increasing with increasing biomass density, average diameter at breast height, leaf area index (LAI), and aboveground net primary productivity (NPP), while sunlit background fraction is decreasing. We show that the end member fractions can be used to estimate biomass with a standard error of $\approx 2 \mathrm{~kg} / \mathrm{m}^{2}$, LAI with a standard error of $0.58, \mathrm{dbh}$ with a standard error of $\approx 2 \mathrm{~cm}$, and aboveground NPP with a standard error of $0.07 \mathrm{~kg} \cdot \mathrm{m}^{-2} \cdot \mathrm{yr}^{-1}$.

We also show that the fraction of sunlit canopy is only weakly correlated with the biophysical variables and are thus able to show why a popular vegetation index, Normalized Difference Vegetation Index (NDVI), does not provide a useful measure of these biophysical characteristics. We do show, however, that NDVI should be related to the fraction of photosynthetically active radiation incident upon and absorbed by the canopy.

This work has convinced us that a paradigm shift in the remote sensing of biophysical characteristics is in order - a shift away from direct inference of biophysical characteristics from vegetation indices and toward a two-step process, in which (1) stand-level reflectance is approximated in terms of linear combinations of reflectance-invariant, spectrally distinct components (spectral end members) and mixture decomposition used to infer the areal fractions of these components, e.g., shadow, sunlit crown, and sunlit background, followed by (2) the use of radiative transfer models to compute biophysical characteristic values as a function of the end member fractions.
\end{abstract}

Key words: biomass; biophysical characteristics; boreal conifers; geometric reflectance models; leaf area index; mixture decomposition; photosynthetically active radiation; Picea mariana; remote sensing.

\section{INTRODUCTION}

Ecosystem process, biosphere-atmosphere transfer, and carbon exchange models all require the parame-

\footnotetext{
${ }^{1}$ Manuscript received 29 March 1994; revised 31 August 1994; accepted 31 August 1994; final version received 23 November 1994.
}

trization of the land surface using vegetation structural characteristics (Bolle 1991, Rasool 1992, Skole 1992, Sellers and Schimel 1993). The required parameters include leaf area index (LAI), biomass areal density, fraction of incident photosynthetically active radiation absorbed by the canopy ( $F$ par), and canopy roughness, $Z_{0}$. Evaporation, gas flux, sensible heat transfer, mo- 
mentum transfer, and precipitation interception are some of the more important quantities that require a knowledge of the above coefficients for their calculation. Although not yet a demonstrated capability, the most feasible method for obtaining these parameters globally, and updating them periodically, is through the use of satellite remote sensing.

A number of studies, beginning in the late 1950s, have explored, both empirically and theoretically, the relationship between pixel-level reflected, absorbed and transmitted radiation, and the associated canopy biophysical characteristics. Measurements at the leaf level (Gates et al. 1965, Gausman and Allen 1973, Gausman 1984), showed that leaf chlorophyll is a strong absorber of light of wavelength $\approx 0.69 \mu \mathrm{m}$ and that leaf cell structures are strong scatterers of light in the near-infrared region of wavelength $\approx 0.8-1.0 \mu \mathrm{m}$. Early investigations (Allen and Richardson 1968) attempted to scale these leaf-level optical properties to the canopy level to explain the reflectance measurements of whole plants. They were able to explain the observed reflectance decrease in the $0.69 \mu \mathrm{m}$ band and the increase in near-infrared reflectance with canopy leaf area by treating the canopy simply as horizontal layers of stacked leaves. Such models predict that leaflevel light absorption by chlorophyll will cause the visible reflectance to decrease exponentially with the number of leaf layers, while the near-infrared reflectance, where scattering predominates, will increase with the number of leaf layers. Such observations and model predictions suggested that remote sensing measures of reflectance in these spectral regions should be useful for inferring canopy-level leaf area index over regional and larger regions.

The central problem in using remotely sensed measures of surface reflectance to infer canopy level structural and biophysical characteristics is the scaling of optical properties from the leaf level, where they can be easily measured and related to leaf composition and structure, to the pixel level, where leaf optics interact with canopy structure, understory characteristics, view, and illumination geometry to produce a complicated relationship among pixel-level reflectance, stand structural, biophysical, and leaf optical properties. In general, when extrapolating leaf-level optical properties to the stand level, the physical assumptions used for horizontal layers of leaves do not hold, yielding unrealistic solutions. Modeling efforts that have addressed this problem are numerous, and can be placed into four general classes of models (Goel 1988): (1) turbid medium models, for examples Suits 1972 and Verhoef 1983, (2) geometric models (Li and Strahler 1985), (3) hybrid combinations of (1) and (2) (Goel and Grier 1988), and (4) complex computer simulation models, for example Goel et al. 1990. These models compute canopy and pixel-level reflectance in terms of not only leaf optical properties, but other biophysical parameters such as overstory and understory leaf area index, leaf angle distribution, bark area index, crown shape, and spacing, etc. The models have been used to infer biophysical characteristics, from pixel-level measures of reflectance of numerical iteration and convergence, i.e., matching reflectance values to parameter sets, a process referred to as "inversion" (Goel and Thompson 1984). The problems with inversion are that (1) the dimensionality of the remote sensing measurement space must equal or exceed the number of parameters being estimated and (2) in the more complex models, the number of parameters that must be estimated is large, and the dimensionality requirements for their "inversion" often exceeds the intrinsic dimensionality of the remotely sensed data. The intrinsic dimensionality of the remotely sensed data for a single viewing angle and date is determined by the number of physically independent (uncorrelated) wave bands, generally no more than three to four. The visible wave band reflectances all respond to the same physical absorption and scattering process by plant pigments, thus these reflectances are highly correlated and do not form a linearly independent set. The near-infrared bands are generally uncorrelated with the visible wavelengths, but near infrared reflectance is largely driven by leaf cell structure and thus different near infrared bands are correlated with each other. Exceptions are the near- and middle-infrared regions where protein, lignin, and starch molecules absorb strongly at certain frequencies. These regions have been the subject of investigation for their ability to provide information on canopy chemical composition (Wessman et al. 1988a, $b$ ). Thermal infrared bands are sensitive primarily to canopy radiative temperature, which is indirectly related to canopy structure, and thus are independent of both visible and reflective infrared; however, their use adds additional parameters to be estimated, parameters related to turbulent heat and long-wave radiative transfer within the canopy. Finally, mid-infrared reflective bands that respond to plant water content are, in live vegetation, highly correlated to plant chlorophyll (Hall 1994). Thus, at most, three to four independent bands are available to estimate the many biophysical parameters that populate complex canopy reflectance models, and two of these, the visible and near-infrared, provide most of the information about canopy structure.

These complications have necessitated the investigation of approaches that hold some of the unknown parameters fixed-estimating them from non-remote sensing data, or approaches that attempt to reduce the influence on reflectance of canopy biophysical parameters that are of little interest to a particular application, for example leaf angle distribution. An alternate approach is to employ vegetation indices that are insensitive to unknown and unwanted variables, while being sensitive to desired parameters. Three frequently cited indices in this vein are in Normalized Difference Vegetation Index (NDVI), or its closely related index, Simple Ratio (SR), and the Kauth-Thomas (KT) greenness 
index (Kauth and Thomas 1976). A number of studies have shown that these indices are sensitive to biomass, LAI, and Fpar (Tucker et al. 1983, Asrar et al. 1984, Sellers 1985), but relatively insensitive to shadowing effects, view, and illumination angles. However, they are also sensitive to understory reflectance (Huete et al. 1985, Goward and Huemmrich 1992), canopy structure, and atmospheric absorption and scattering.

It is known that stand-level reflectance for canopies exhibiting distinct geometric features, such as conifers, is strongly related to shadow fraction, sunlit canopy fraction, sunlit background fraction, and their reflectance (Li and Strahler 1985, Jasinski 1990). Li and Strahler performed Monte Carlo simulations, randomly placing cones within a pixel over a snow background, to examine the relationships between pixel-level KT greenness and brightness and the fraction of illuminated cone area, fraction of illuminated background area, and shadow area. They showed that as canopies were randomly added to the pixel field of view, the KT greenness-brightness followed a two-dimensional trajectory, originating in the illuminated bare snow point and terminating at a different point in the KT space, greenness-brightness value of which was defined by the average reflectance of sunlit and shadowed canopy. The shape of this trajectory and the position along it were uniquely determined by the height : width ratio of the canopy, the number of canopies, and the reflectance of the sunlit canopy, shadows, and the sunlit background. Thus, they were able to "invert" or solve their geometric model using, as input, multispectral measures of KT greenness and brightness for (1) the number of canopies within a pixel, (2) the average height of the canopies, and (3) the average cone angle for the canopies. They applied this inversion technique to KT greenness and brightness data collected from red fir canopies, but found that the errors in determining the canopy parameters were large, ranging up to $\pm 100 \%$.

The dependence of NDVI on canopy fraction has been investigated (Jasinski 1990) using a simple geometric shadowing model consisting of randomly placed opaque blocks within a pixel. He assigned arbitrary reflectance values to the blocks, the background, and the shadows and showed that NDVI is a monotonically increasing function of the fraction of sunlit canopy cover within the pixel, and is strongly sensitive to background reflectance. He compared the NDVI predictions from this model to actual NDVI measurements taken over a pecan orchard and juniper forests, and showed that indeed, the predicted NDVI increase with canopy cover was observed.

Finally, Rosema et al. (1992) have extended the work of Li and Strahler (1985) and Jasinski and Eagleson (1989) to develop a forest canopy-light interaction model in which the forest is viewed as a discontinuous canopy layer with crowns and gaps, and apply this model to estimate forest biophysical parameters for Scotch pine in the Netherlands. They modeled forest stand reflectance as linear combinations of the areal fractions of four radiometrically active elements: (1) shadowed and (2) sunlit background as viewed through a canopy; (3) shadowed and (4) sunlit background viewed directly, unobscured by canopy. The fractions of these four elements viewed by the sensor were in turn modeled as functions of biophysical parameters: tree density and crown horizontal area as well as canopy LAI, view and illumination angle, crown height and crown geometry, and leaf angle distribution. These biophysical parameters were then in turn estimated by inversion of the model using satellite-measured reflectances in a two-step process: first, the proportions of the radiometrically active elements were estimated from measured reflectance in the Landsat Thematic Mapper (TM) near-infrared band 4 and the TM midinfrared band 5 . This is accomplished by simultaneous solution of two linear equations (given estimates of reflectances of an infinitely deep canopy and background) that express the stand-level reflectances in these bands as functions of (1) the fraction of infinitely deep canopy and (2) the fraction of shadowed and sunlit background within a stand. Infinitely deep canopy reflectance values are obtained from TM observations of the densest canopies within the scene, and "ground" reflectance values from TM observations of clear-cut areas. Using this approach, the authors did not find very high correlations with existing ground data, and speculate that the poor correlations are "... partly caused by the low accuracy of the ground data."

The initial developments of our approach were done independently of the work of Rosema et al. (1992); however, we became aware of their paper as we were preparing our own for submission, and recognized that the biophysical parameter estimation we had developed, and report herein, is similar in some respects to theirs. We use a simpler canopy reflectance model with fewer parameters and somewhat different radiometrically active fractions. Further, we do not attempt a direct inversion to obtain the biophysical parameters. We do obtain significant and useful correlations between TM reflectance in two bands and biophysical parameters. We are also able to show directly why NDVI is not well correlated with forest structural parameters, but is useful for estimating photosynthetically active radiation absorbed by the canopy.

In our work, we were interested in taking a somewhat difference route to biophysical parameter estimation than using vegetation indices to infer biophysical parameters. Our evaluations of vegetation indices, such as NDVI and KT greenness to estimate biophysical parameters for boreal coniferous forests, had not been fruitful. Biomass or LAI showed very little correlation with these remote sensing indices. As an alternative, we decided to utilize the individual band reflectances in the red and near-infrared to first infer the proportion of a pixel occupied by sunlit crown, shadows, and sunlit background, then relate these intermediate variables to 
TABLE 1. Overstory composition of black spruce sites in the Superior National Forest based on fractional stem count of trees $>2 \mathrm{~m}$ in height. See Table 4 for key to species' abbreviations.

\begin{tabular}{ccccc}
\hline \hline & \multicolumn{4}{c}{ Species } \\
\cline { 2 - 5 } Site & PIMA & LALA & PIBA & PIST \\
\hline \multicolumn{4}{c}{ Relative composition } \\
2 & 1.00 & 0.00 & 0.00 & 0.00 \\
12 & 0.88 & 0.12 & 0.00 & 0.00 \\
14 & 1.00 & 0.00 & 0.00 & 0.00 \\
15 & 1.00 & 0.00 & 0.00 & 0.00 \\
18 & 0.94 & 0.06 & 0.00 & 0.00 \\
19 & 0.98 & 0.02 & 0.00 & 0.00 \\
38 & 0.95 & 0.01 & 0.04 & 0.00 \\
39 & 0.97 & 0.01 & 0.01 & 0.00 \\
41 & 1.00 & 0.00 & 0.00 & 0.00 \\
42 & 0.97 & 0.00 & 0.00 & 0.00 \\
43 & 0.99 & 0.00 & 0.00 & 0.01 \\
49 & 0.91 & 0.00 & 0.08 & 0.00 \\
50 & 1.00 & 0.00 & 0.00 & 0.00 \\
51 & 0.95 & 0.05 & 0.00 & 0.00 \\
52 & 1.00 & 0.00 & 0.00 & 0.00 \\
55 & 1.00 & 0.00 & 0.00 & 0.00 \\
63 & 0.87 & 0.12 & 0.00 & 0.00 \\
Average & 0.96 & 0.02 & 0.01 & 0.00 \\
\hline
\end{tabular}

the canopy biophysical parameters of interest. The motivation was twofold. First, we believed that mixture decomposition techniques in the vein of Horowitz et al. (1971) might be capable of decomposing boreal conifer scenes into corresponding proportions of shadow, sunlit background, and sunlit canopy, i.e., the "end member" reflectances. Our reflectance measurements for boreal coniferous species (Hall et al. 1992) demonstrated that the visible and near-infrared spectral contrast among these end members is quite large, a necessary condition to robust mixture decomposition. Secondly, the results from other geometric optics models
(Li and Strahler 1985) suggested that the biophysical characteristics might be strongly related to canopy cover and shadow fraction. If true, these two factors would provide an alternate approach to estimating canopy biophysical characteristics for an overwhelming portion of the boreal ecosystem.

We report in this paper, the development and evaluation of such an approach. We begin by describing the data set used, then proceed to outline the analysis approach for mixture decomposition, and the approach for estimation of the "end member" reflectances. Finally, we present the results of this analysis and draw a number of conclusions regarding future directions.

\section{The Data Set}

During the summers of 1983 and 1984 a multispectral and biophysical characteristics data set was acquired over an $\approx 40 \times 120 \mathrm{~km}$ study site in the Superior National Forest (SNF) near Ely, Minnesota. During this field experiment, called COVER (Characterization of Vegetation with Remote Sensing, described in Hall et al. 1992), more than $80 \approx 80 \times 80 \mathrm{~m}$ plots containing the boreal dominants black spruce (Picea" mariana), jack pine (Pinus banksiana), and trembling aspen (Populus tremuloides), were ground-visited throughout the area. The overstory and understory vegetation was inventoried and relevant biophysical characteristics estimated, such as community composition and structure, biomass density, stem density, diameter at breast height, net primary productivity, etc. Table 1 lists, for the sites used in this present study, the relative composition of the overstory based on fractional stem count for trees $>2 \mathrm{~m}$ in height. Table 2 contains similar information for the understory on each site.

The approach to obtaining quantitative estimates of

TABle 2. Understory composition of black spruce sites in the Superior National Forest. Percentages are the average of five $2 \mathrm{~m}$ diameter subsamples in each site. Species with less than an average of $1 \%$ cover over all sites are not shown.

\begin{tabular}{|c|c|c|c|c|c|c|c|c|c|c|c|c|c|c|c|c|c|c|c|c|c|c|}
\hline \multirow{2}{*}{$\begin{array}{l}\text { Spe- } \\
\text { cies* }\end{array}$} & \multicolumn{22}{|c|}{ Site } \\
\hline & 2 & 12 & 14 & 15 & 18 & 19 & 38 & 39 & 41 & 42 & 43 & 45 & 47 & 48 & 49 & 50 & 51 & 52 & 54 & 55 & 56 & 57 \\
\hline & \multicolumn{22}{|c|}{ Ground cover $(\%)$} \\
\hline SPHA & 68 & 60 & 16 & 68 & 62 & 62 & 82 & 60 & 72 & 34 & 32 & 55 & 64 & 64 & 48 & 38 & 74 & 86 & 62 & 38 & 68 & 72 \\
\hline LEGR & 33 & 5 & 14 & 27 & 6 & 6 & 24 & 24 & 6 & 15 & 28 & 7 & 9 & 6 & 1 & 11 & 34 & 36 & 22 & 4 & 34 & 17 \\
\hline MOSS & 3 & 0 & 72 & 30 & 3 & 0 & 10 & 34 & 4 & 36 & 34 & 8 & 5 & 13 & 6 & 38 & 2 & 5 & 0 & 18 & 6 & 4 \\
\hline BLIT & 14 & 1 & 14 & 7 & 7 & 5 & 10 & 3 & 17 & 14 & 13 & 25 & 8 & 12 & 5 & 18 & 3 & 3 & 16 & 30 & 13 & 5 \\
\hline CHCA & 5 & 9 & 1 & 8 & 12 & 28 & 12 & 0 & 0 & 1 & 3 & 4 & 8 & 0 & 0 & 0 & 17 & 9 & 10 & 2 & 5 & 20 \\
\hline SEDG & 0 & 1 & 0 & 0 & 0 & 0 & 0 & 0 & 0 & 5 & 0 & 10 & 32 & 2 & 14 & 6 & 0 & 1 & 0 & 2 & 5 & 14 \\
\hline SMTR & 14 & 14 & 13 & 12 & 0 & 0 & 6 & 9 & 5 & 6 & 6 & 4 & 20 & 10 & 5 & 4 & 2 & 0 & 7 & 6 & 2 & 0 \\
\hline GAHI & 28 & 2 & 7 & 13 & 0 & 0 & 2 & 4 & 6 & 8 & 5 & 3 & 4 & 1 & 2 & 3 & 0 & 4 & 3 & 6 & 3 & 4 \\
\hline GRAS & 5 & 12 & 2 & 4 & 4 & 1 & 8 & 5 & 3 & 0 & 0 & 0 & 1 & 10 & 5 & 2 & 1 & 17 & 5 & 0 & 0 & 0 \\
\hline PIMA & 1 & 3 & 2 & 3 & 13 & 15 & 2 & 6 & 1 & 4 & 2 & 0 & 2 & 1 & 0 & 0 & 6 & 1 & 1 & 4 & 6 & 3 \\
\hline VAOX & 6 & 4 & 1 & 3 & 5 & 5 & 1 & 4 & 0 & 2 & 1 & 2 & 5 & 0 & 1 & 1 & 3 & 2 & 2 & 2 & 4 & 4 \\
\hline VAAN & 4 & 0 & 5 & 5 & 0 & 0 & 4 & 5 & 5 & 9 & 5 & 5 & 5 & 0 & 2 & 0 & 0 & 0 & 4 & 5 & 2 & 0 \\
\hline ERIO & 0 & 3 & 0 & 0 & 11 & 10 & 0 & 0 & 0 & 0 & 0 & 0 & 0 & 0 & 0 & 0 & 8 & 0 & 0 & 0 & 0 & 0 \\
\hline FUNG & 1 & 4 & 0 & 4 & 1 & 0 & 1 & 0 & 0 & 0 & 0 & 0 & 3 & 3 & 0 & 0 & 0 & 1 & 0 & 0 & 4 & 3 \\
\hline KAPO & 0 & 3 & 1 & 0 & 2 & 5 & 1 & 1 & 0 & 0 & 0 & 1 & 1 & 0 & 0 & 0 & 5 & 0 & 0 & 0 & 2 & 2 \\
\hline GLIT & 2 & 0 & 0 & 0 & 0 & 0 & 3 & 1 & 3 & 8 & 2 & 5 & 3 & 4 & 0 & 0 & 0 & 1 & 4 & 0 & 0 & 0 \\
\hline
\end{tabular}

* See Table 4 for key to abbreviations.

$\dagger$ Average for all sites. 
the overstory biophysical characteristics, shown in Table 3 , involved allometric analysis of sacrificed trees (31 black spruce and 32 aspen) to relate convenience measurements such as diameter at breast height and branch diameter to the biophysical characteristics of interest such as bole and branch biomass. Relationships between these variables developed for the sacrificed trees were then applied to the convenience measurements at the individual tree level within the sample plots, then aggregated over all trees in the plots to compute site biophysical characteristics. Net primary productivity (NPP) was estimated from allometry, relating bole NPP to the average radial growth over 5 years as determined from bole segments. Terminal growth was estimated from observed increases in tree height over those same years. The allometric equations were used to back-project bole biomass five years and determine biomass at the earlier period. The change in total bole biomass and terminal growth over the 5-yr interval was used as an estimate of aboveground productivity. The details of the dimension analysis method can be found elsewhere (Woods et al. 1985, 1991). Table 4 provides abbreviation names used in Tables 1-3.

Using a helicopter-mounted Barnes Modular Multiband Radiometer (MMR) from an altitude of from 120 to $180 \mathrm{~m}$ above ground, spectral reflectance measurements were acquired over each of the ground-visited plots at several times during the 1983 and 1984 growing seasons. The field of view of the MMR was adjusted with flight altitude to maintain an $\approx 30 \times 30 \mathrm{~m}$ pixel on the ground. The spectral reflectance data were calibrated but not corrected for atmospheric effects, which are relatively small at this altitude. This data set, as well as other data collected during COVER, has been archived in electronic form and documented in detail (Hall et al. 1992).

TABle 2. Continued.

\begin{tabular}{rrrrrrrrrr}
\hline \hline \multicolumn{10}{c}{ Site } \\
\hline 62 & 63 & 64 & 68 & 100 & 101 & 102 & 103 & 105 & Avg* \\
\hline \multicolumn{10}{c}{ Ground cover (\%) } \\
66 & 56 & 56 & 62 & 60 & 36 & 50 & 60 & 70 & 58 \\
7 & 12 & 6 & 4 & 0 & 0 & 8 & 10 & 6 & 14 \\
12 & 1 & 6 & 9 & 10 & 10 & 10 & 9 & 16 & 13 \\
1 & 8 & 0 & 8 & 17 & 35 & 8 & 2 & 9 & 11 \\
20 & 12 & 24 & 5 & 0 & 0 & 4 & 15 & 0 & 7.5 \\
6 & 0 & 34 & 10 & 20 & 1 & 6 & 26 & 11 & 6.6 \\
0 & 0 & 0 & 1 & 2 & 0 & 2 & 1 & 3 & 5 \\
0 & 0 & 0 & 5 & 2 & 1 & 4 & 1 & 3 & 4 \\
17 & 6 & 0 & 1 & 0 & 0 & 6 & 0 & 2 & 3.8 \\
4 & 8 & 7 & 1 & 0 & 0 & 3 & 8 & 3 & 3.5 \\
4 & 3 & 4 & 5 & 1 & 2 & 4 & 5 & 5 & 2.9 \\
0 & 0 & 0 & 0 & 1 & 0 & 0 & 0 & 0 & 2.1 \\
0 & 24 & 0 & 0 & & & & & & 1.8 \\
0 & 0 & 0 & 2 & 5 & 2 & 3 & 3 & 5 & 1.5 \\
1 & 8 & 4 & 2 & 0 & 0 & 1 & 5 & 0 & 1.5 \\
0 & 0 & 0 & 0 & & & & & & 1.2 \\
\hline
\end{tabular}

As can be seen in Table 1, the sites used in this study are $>90 \%$ black spruce, with the exception of sites 12 and 63 which have significant fractions of larch (Larix laricina). Stands ranged from very sparse on wet, nutrient-poor bogs, to dense, closed stands on drier, more productive peat lands. As can be seen in Table 2, a common feature to the stands was dominant mats of sphagnum moss (Sphagnum spp.), populated by Labrador tea (Ledum groenlandicum), other shrubs, and miscellaneous grasses and sedges common to wet, poorly drained soils.

\section{Data Analysis}

The data analysis consists of three main computations: (1) modeling pixel-level reflectance in terms of the reflectance of fundamental scene elements, or end members, i.e., shadow, sunlit canopy, and sunlit background; (2) measurement and estimation of the end member's reflectances, and (3) mixture decomposition for inference of the areal proportions of these scene elements from measured pixel-level reflectance values.

\section{Modeled pixel-level reflectance values}

Fig. 1 shows the red (of wavelength $0.63-0.69 \mu \mathrm{m}$ ) versus the infrared (of wavelength $0.75-0.88 \mu \mathrm{m}$ ) stand-level reflectance for the SNF black spruce stands acquired at nadir by the helicopter-mounted MMR. The data were acquired over a 2 -yr period for a range of solar illumination angles $\left(27^{\circ}-75^{\circ}\right)$, as described in the previous section. Plotted also in Fig. 1 are the measured reflectances of the sphagnum background of the black spruce stands, the reflectance for a deep, clear lakea surrogate for shadow reflectance, and inferred sunlit canopy reflectance. The inference of sunlit canopy reflectance will be discussed in the section to follow.

To model the shape of the scatter gram in Fig. 1, we assume that radiative transfer among the various vegetative components in the overstory and understory (needles, twigs, branches, shrubs, etc.) can be greatly simplified and represented by a linear process where pixel-level reflectance can be computed as a linear combination of bulk component reflectances: sunlit canopy, sunlit background (or understory), and shadow reflectance. More precisely,

$$
\rho_{t}=C \rho_{c}+S \rho_{s}+B \rho_{b}
$$

where

$\rho_{t}=$ pixel-level reflectance,

$\rho_{c}=$ sunlit canopy reflectance,

$\rho_{s}=$ shadowed canopy and shadowed background reflectance,

$\rho_{b}=$ background reflectance,

$C=$ sunlit canopy fraction,

$S=$ fraction of shadow (canopy plus background), and

$B=$ fraction of sunlit background.

To achieve this linearization of the radiative transfer 
TABLE 3. Site-averaged overstory biophysical characteristics for the Superior National Forest. Data represent average of five $16 \mathrm{~m}$ diameter subplots in $80 \times 80 \mathrm{~m}$ sites.

\begin{tabular}{|c|c|c|c|c|c|c|}
\hline \multirow[b]{2}{*}{ Site } & \multicolumn{6}{|c|}{ Biophysical characteristic } \\
\hline & $\mathrm{dbh}(\mathrm{cm})$ & $\begin{array}{l}\text { Stem density } \\
\left(\text { no. } / \mathrm{m}^{2}\right)\end{array}$ & Basal fraction* & $\begin{array}{l}\text { Biomass density } \\
\left(\mathrm{kg} / \mathrm{m}^{2}\right)\end{array}$ & $\begin{array}{c}\mathrm{NPP} \\
\left(\mathrm{kg} / \mathrm{m}^{2}\right)\end{array}$ & $\mathrm{LAI} \dagger$ \\
\hline 2 & 14.52 & 0.175 & 0.00317 & 12.38 & 0.325 & 2.88 \\
\hline 12 & 4.54 & 0.164 & 0.00032 & 0.68 & 0.039 & 0.48 \\
\hline 14 & 13.22 & 0.247 & 0.00372 & 13.64 & 0.432 & 3.27 \\
\hline 15 & 12.21 & 0.224 & 0.00288 & 10.68 & 0.348 & 2.69 \\
\hline 18 & 4.24 & 0.261 & 0.00046 & 1.09 & 0.063 & 0.74 \\
\hline 19 & 4.05 & 0.256 & 0.00041 & 1.03 & 0.058 & 0.69 \\
\hline 38 & 7.33 & 0.470 & 0.00240 & 6.79 & 0.295 & 2.69 \\
\hline 39 & 5.15 & 0.377 & 0.00097 & 2.37 & 0.118 & 1.32 \\
\hline 41 & 13.49 & 0.183 & 0.00308 & 11.14 & 0.349 & 2.84 \\
\hline 42 & 8.60 & 0.259 & 0.00218 & 7.31 & 0.258 & 2.28 \\
\hline 43 & 8.44 & 0.363 & 0.00268 & 8.70 & 0.330 & 2.79 \\
\hline 45 & 7.28 & 0.534 & 0.00278 & 8.45 & 0.358 & 3.09 \\
\hline 47 & 5.29 & 0.582 & 0.00156 & 3.53 & 0.180 & 2.00 \\
\hline 48 & 9.83 & 0.340 & 0.00285 & 9.15 & 0.404 & 2.70 \\
\hline 49 & 7.38 & 0.712 & 0.00360 & 10.09 & 0.410 & 3.74 \\
\hline 50 & 7.61 & 0.676 & 0.00369 & 10.36 & 0.432 & 3.73 \\
\hline 51 & 5.92 & 0.378 & 0.00131 & 3.62 & 0.174 & 1.69 \\
\hline 52 & 9.92 & 0.356 & 0.00318 & 10.04 & 0.375 & 3.03 \\
\hline 54 & 5.71 & 0.552 & 0.00193 & 5.57 & 0.267 & 2.44 \\
\hline 55 & 7.56 & 0.529 & 0.00287 & 8.58 & 0.360 & 3.09 \\
\hline 56 & 8.09 & 0.256 & 0.00168 & 5.28 & 0.215 & 1.83 \\
\hline 57 & 9.52 & 0.315 & 0.00262 & 8.29 & 0.319 & 2.60 \\
\hline 62 & 4.52 & 0.196 & 0.00039 & 0.89 & 0.051 & 0.59 \\
\hline 63 & 3.94 & 0.319 & 0.00051 & 1.27 & 0.071 & 0.84 \\
\hline 64 & 5.09 & 0.148 & 0.00035 & 0.88 & 0.049 & 0.52 \\
\hline 68 & 7.40 & 0.660 & 0.00325 & 8.72 & 0.382 & 3.48 \\
\hline 100 & 10.98 & 0.455 & 0.00455 & 15.05 & 0.538 & 4.00 \\
\hline 101 & 7.22 & 1.074 & 0.00487 & 13.50 & 0.572 & 5.42 \\
\hline 102 & 5.92 & 0.917 & 0.00292 & 7.25 & 0.346 & 3.67 \\
\hline 103 & 4.86 & 0.200 & 0.00050 & 1.35 & 0.066 & 0.71 \\
\hline 105 & 10.48 & 0.459 & 0.00447 & 15.14 & 0.538 & 4.26 \\
\hline
\end{tabular}

* Basal fraction is the ratio of bole area to surface area.

$\dagger$ LAI is the projected leaf area index.

equations requires that the aggregate component reflectances are constant, and thus do not depend on their relative areal abundances. This assumes, for example, that sunlit canopy reflectance does not depend on sunlit canopy fraction; hence the canopy and stand density.
These assumptions of course do not hold exactly, but if they do hold approximately, they permit a very useful simplification of an enormous problem. Furthermore, we have in the data set of Fig. 1 the ability to evaluate this simplifying assumption.

TABLE 4. Abbreviation names used in Tables 1-3.

\begin{tabular}{lll}
\hline \hline Abbreviation & \multicolumn{1}{c}{ Common name } & \multicolumn{1}{c}{ Scientific name } \\
\hline BLIT & Brown litter & \\
CHCA & Leatherleaf & Chamaedaphne calyculata \\
ERIO & Cotton grass & Eriophorum spp. \\
FUNG & Fungi & Gaultheria hispidula \\
GAHI & Creeping strawberry & \\
GLIT & Green litter & \\
GRAS & Grasses & Kalmia polifolia \\
KAPO & Bog laurel & Larix laricina \\
LALA & Tamarack (larch) & Ledum groenlandicum \\
LEGR & Labrador tea & \\
MOSS & Mosses (non-sphagnum) & Pinus banksiana \\
PIBA & Jack pine & Picea mariana \\
PIMA & Black spruce & Pinus strobus \\
PIST & White pine & Smilacina trifoliata \\
SEDG & Sedges & Sphagnum spp. \\
SMTR & Bog false Solomon seal & Vaccinium angustifolium \\
SPHA & Sphagnum moss & Vaccinium oxycoccus \\
VAAN & Lowbush blueberry & \\
VAOX & Small cranberry & \\
\hline
\end{tabular}


We do assume that the bulk component fractions interact with each other. To compute these interactions, we use the approximation of Jasinski and Eagleson (1989) and Jasinski and Eagleson (1990), who represent canopies as randomly spaced vertical cylinders. The fraction of background that is shadowed by the cylinders is given by them as:

$$
S=1-C-(1-C)^{(\eta+1)} .
$$

$\eta$ is calculated using the height to width ratio of the cylinder, $R$, and the solar zenith angle, $\theta$ :

$$
\eta=R \tan (\theta)
$$

Thus, $S$ will vary with cylinder diameter and height and the solar zenith angle. $C$ is the sum of the crosssectional areas of the non-overlapping cylinders divided by the pixel area and $B$ is:

$$
B=1-C-S \text {. }
$$

Thus, using Eqs. 2-4 we may compute how shadow and sunlit background fraction depend on sunlit canopy fraction and solar zenith angle (SZA). Fig. 2 shows the predicted values of $S$ from Eq. 2 for an $R$ of 7. This $R$ value is based on observations of spruce trees in the SNF and does not seem to vary greatly among stands. The calculations of $C, B$, and $S$ are for a nadir view only, although they can be extended to off-nadir view angles for other sensors such as the Advanced Very High Resolution Radiometer (AVHRR) aboard the National Oceanic and Atmospheric Administration (NOAA) series of satellites or the High Resolution Visible (HRV) instruments aboard Systeme Probatoire pour "Observation de la Terre" (SPOT). As can be observed from Fig. 2, shadow fraction increases with sunlit canopy fraction until a critical value for sunlit canopy fraction is reached, then shadow fraction begins to decrease. The sunlit background fraction decreases

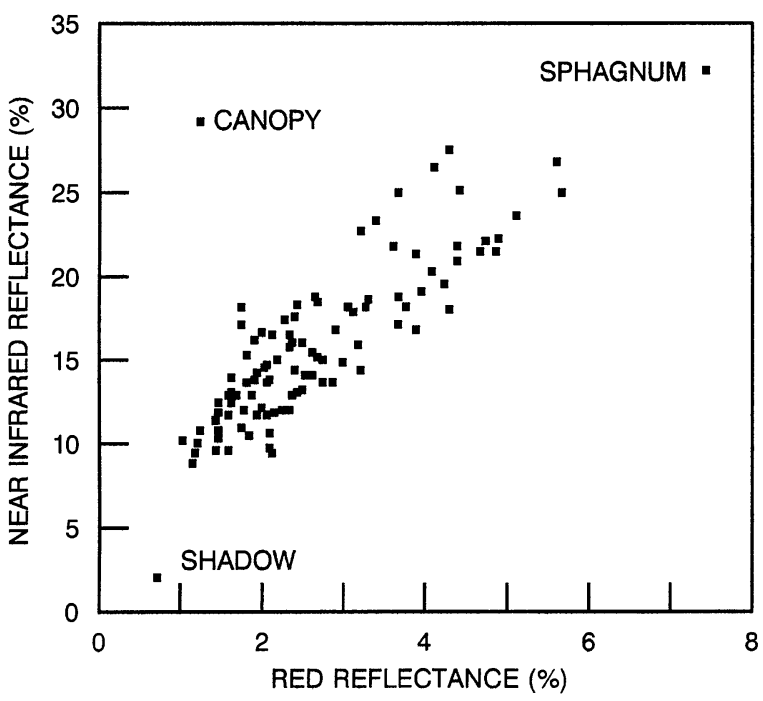

FIG. 1. Near-infrared versus red reflectance for 31 black spruce stands as acquired by the helicopter-mounted MMR.

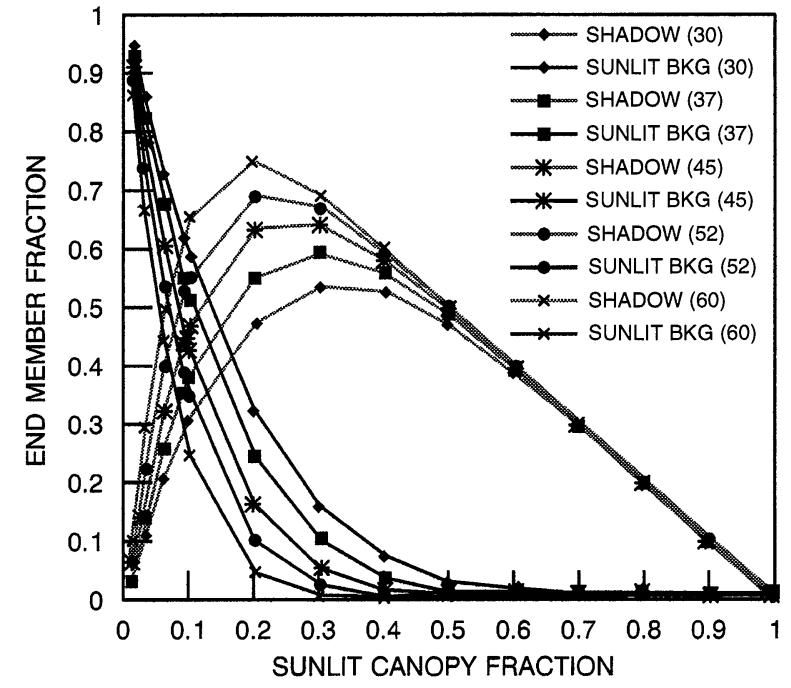

FIG. 2. Predicted values of shadow and sunlit background fraction from the cylindrical canopy model plotted against sunlit canopy fraction for solar zenith angles (SZAs) of $30^{\circ}$, $37^{\circ}, 45^{\circ}, 52^{\circ}$, and $60^{\circ}$ (SZA value in parenthesis in key).

monotonically with sunlit canopy fraction. The rates at which $S$ and $B$ vary with $C$ depend on SZA as a function of the geometry of shadowing. As can be seen from Eq. 1, the relative rate of change of $S$ with respect to $B$ is pivotal to understanding the shape of the Fig. 1 scatter gram. We will explore this more fully after we have obtained values for the end member reflectances in Eq. 1.

\section{End member reflectances}

The assumption that each of the bulk canopy components have constant reflectance permits us to define the concept of "end member" reflectance. The concept of end member reflectance derives from the location of the red and near-infrared reflectances of the canopy components as displayed in Figs. 1 and 3. All other pixel values lie within a triangle whose vertices are defined by the end member reflectance values. Except for sphagnum moss, we did not have direct measures of the end member reflectances.

Obtaining values for shadow and sunlit canopy reflectances required both measurement and inference from measurement. We had hand-held measurements of the sunlit sphagnum background reflectance $\rho_{b}$. The assumption that $\rho_{b}$ is constant across all sites is reasonable since the understories of the black spruce stands were predominantly sphagnum moss (see Table 2 ). To obtain a value for $\rho_{s}$, the shadowed background and shadowed canopy reflectance, we assumed that they are roughly equal to the reflectance of deep lakes in the area, for which we also had measurements. $\rho_{s}$ could also have been calculated using radiative transfer calculations to estimate the amount of light transmitted through the canopy or scattered onto the background from the atmosphere or adjacent canopies. $\rho_{s}$ would be 


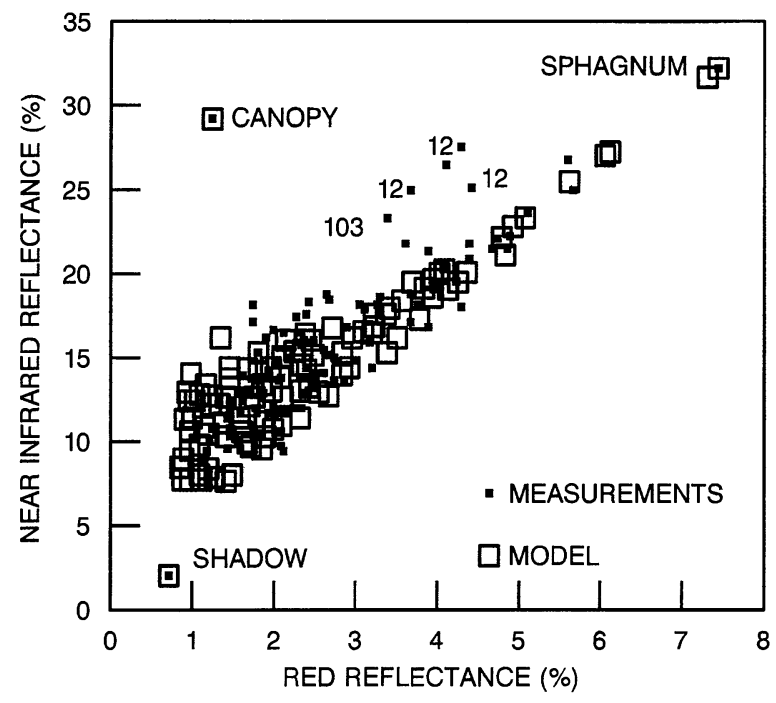

FIG. 3. Comparison of measured and modeled red and near-infrared reflectances for 31 black spruce stands. Measurements were acquired by a helicopter-mounted MMR during the summers of 1983 and 1984 in the Superior National Forest. Modeled reflectances are as described in the text. Numbered points are site identifiers in Table 1.

the amount of light transmitted or scattered onto the background, multiplied by the illuminated background reflectance. Sensitivity studies indicate that small variations in the shadowed background reflectance are not critical to the overall calculations because the shadows are quite dark relative to the canopy and background reflectances. We will take this up in more detail in the discussion section. To estimate $\rho_{c}$, the sunlit canopy reflectance, we solved Eq. 1 for $\rho_{c}$ at the maximum shadow point, assuming that the minimum values of red and near-infrared (NIR) reflectance in Fig. 1 occur at maximum shadow, using the values for $\rho_{s}$, and $\rho_{b}$ discussed directly above. We solved Eq. 1 for several ranges of SZA: $27^{\circ}-33^{\circ}, 42^{\circ}-48^{\circ}, 49^{\circ}-55^{\circ}$, and $61^{\circ}-$ $67^{\circ}$. The value for $\rho_{c}$ varied among different SZAs. However, by solving this over determined system of equations for the four different SZA ranges using a least squares approach as described in Shimabukuro (1987), we obtained a "best estimate" of $\rho_{c}$. The values for end member reflectances so obtained are given in Table 5 .

\section{Pixel-level reflectance calculations}

To predict the pixel-level value of reflectance as end member proportions vary, we combine Eqs. 1-4, using the end member reflectances as computed as described immediately above. We assume that the end member reflectances do not vary with end member proportions. This assumption is a linear approximation, which ignores interactions among end member proportions and their reflectances. For example, multiple scattering between the canopy and the understory can affect shadow reflectance, which will in turn depend on $C$ and $S$ in
TABLE 5. End member reflectances as derived from measurement and modeling.

\begin{tabular}{lccc}
\hline \hline $\begin{array}{c}\text { Reflectance } \\
\text { (spectral region) }\end{array}$ & $\begin{array}{c}\text { Sunlit } \\
\text { background } \\
(\%)\end{array}$ & $\begin{array}{c}\text { Sunlit } \\
\text { vegetation } \\
(\%)\end{array}$ & $\begin{array}{c}\text { Shadow } \\
(\%)\end{array}$ \\
\hline Red & 7.45 & 1.26 & 0.74 \\
Near-Infrared & 32.1 & 29.22 & 2.2 \\
\hline
\end{tabular}

Eq. 1. $\rho_{c}$ certainly depends to some degree on $C$. Introducing such interactions will likely improve model accuracy to some degree; however, it will complicate the mixture decomposition algorithm since Eq. 1 will no longer be linear.

We will refer to our linear approximation as the cylindrical canopy model. Fig. 3 shows the predicted reflectance of the spruce stands using the cylindrical canopy model in comparison to the measured values. Here we see both the visible and NIR reflectance decreasing from the bright sphagnum end member value as canopy density begins to increase, increasing both the fraction of sunlit crown and shadow. The visible and near-infrared reflectances continue to decrease, colinearly, until a critical value of crown closure is reached. This is the point of maximum shadow shown in Fig. 2. At this point, the crown closure of the canopy becomes sufficiently large so that background shadows themselves are obscured by sunlit canopy, driving the reflectance values toward the sunlit canopy end member reflectance value as sunlit canopy density increasingly obscures the shadowed background. We will refer to the crown closure value at which both red and near-infrared reflectance begins to increase with crown closure as the critical density. The critical density value, a function of SZA, will play an important role in using reflectance to infer biophysical characteristics.

Five of the data points are significantly different than the model predictions. A check of the data reveals that three of the data points are measurements of site 12 with an unusual amount of larch (Larix laricina), as shown in Table 1, and two are from a low density site with an unusual amount of sedge cover in the background, as shown in Table 2. Larch has a different spectral signature than black spruce, and sedges are spectrally different from sphagnum; thus, the end member reflectances are different. We believe these differences explain the lack of fit between the model and the measured reflectance for these points.

\section{Mixture decomposition}

The idea that pixel reflectance is a linear combination of the reflectances of end member components such as vegetation, soil, etc. was introduced over two decades ago (Horowitz et al. 1971). This approach was initially applied to resolve pixel mixtures of cultural vegetation such as corn and soybeans, but was only marginally successful because the reflectance differences between one vegetation type and the next is not sufficient to 

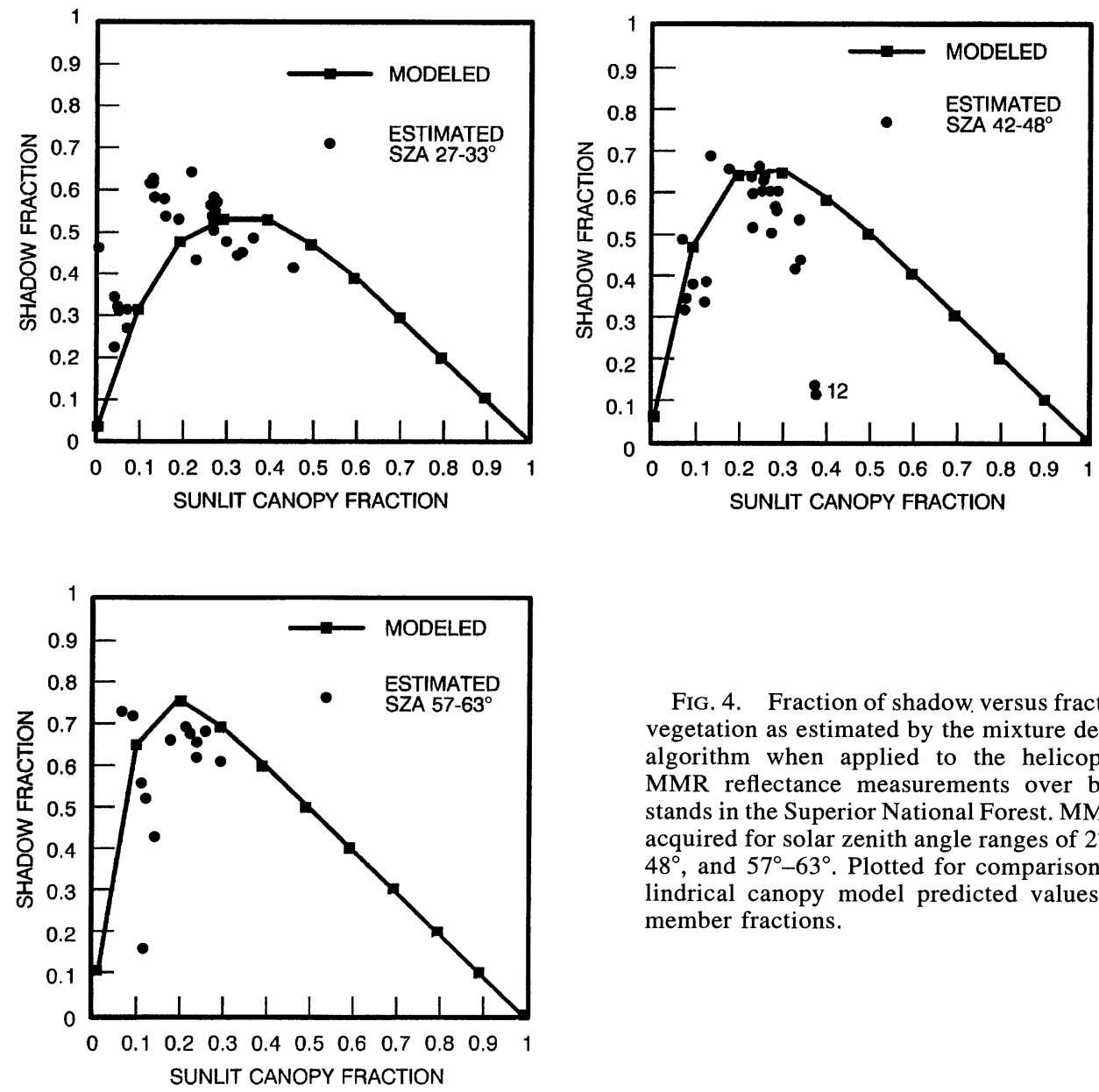

reliably estimate mixture fractions. In the early 1990s, the concept was revived and used to estimate the mixtures of vegetation, soil, and shadow over desert ecosystems (Smith et al. 1990a, $b$, Ustin et al. 1993). In vector notation, pixel reflectance is given by

$$
\boldsymbol{r}=A \boldsymbol{x}+\boldsymbol{e}
$$

Where:

$$
\begin{aligned}
\boldsymbol{r}= & \text { an } n \text {-dimensional reflectance vector for a pixel } \\
& \text { whose components are the measured reflec- } \\
& \text { tances in } n \text { spectral bands, } \\
A= & \text { spectral response matrix }- \text { the } i \text { th and } j \text { th row- } \\
& \text { column element is the reflectance in band } i \text { of } \\
& \text { end member } j, \\
\boldsymbol{x}= & \text { areal fraction of pixel occupied by end mem- } \\
& \text { ber } j, \text { and } \\
\boldsymbol{e}= & \text { error. }
\end{aligned}
$$

Given that $\boldsymbol{r}$ is known for at least $n$ independent spectral bands, and the elements of $A$ are known (equivalent to knowing the reflectances of all end members), Eq. 5 can be inverted for $\boldsymbol{x}$, minimizing the error $\boldsymbol{e}^{\boldsymbol{t}}$. Because the areal fraction of the sum of the end member fractions must be unity, an additional constraint is imposed, namely:

$$
\begin{gathered}
|x|=1_{\text {Results }} \quad 0 \leq x_{i} \leq 1 . \\
\text { Re }
\end{gathered}
$$

FIG. 4. Fraction of shadow, versus fraction of sunlit vegetation as estimated by the mixture decomposition algorithm when applied to the helicopter-mounted MMR reflectance measurements over bläck spruce stands in the Superior National Forest. MMR data were acquired for solar zenith angle ranges of $27^{\circ}-33^{\circ}, 42^{\circ}-$ $48^{\circ}$, and $57^{\circ}-63^{\circ}$. Plotted for comparison are the cylindrical canopy model predicted values of the end member fractions.

\section{Mixture decomposition of helicopter MMR data}

Figs. 4 and 5 show the shadow fraction and the sunlit background fraction plotted against the estimated sunlit vegetation fraction for several different solar illumination angles. These fractions have been estimated from the helicopter MMR data using the mixture decomposition algorithm. Plotted for comparison are the fractions as predicted by the cylindrical canopy model. The computed fractions correspond, with some scatter, to the extracted mixture fractions.

In Fig. 4, for example, the empirical estimates of shadow fraction increase with empirical estimates of sunlit crown fraction until about a quarter to a third of the pixel is sunlit canopy. Then the shadow fraction begins to decrease as shadows are increasingly obscured by sunlit canopy. This behavior is observed at all solar zenith angles. As can be seen from Fig. 4, the critical density value progressively decreases with solar zenith angle increase. This is a result of the fact that larger SZAs lead to higher values of shadow fraction and therefore, a more rapid obscuration of shadows by 

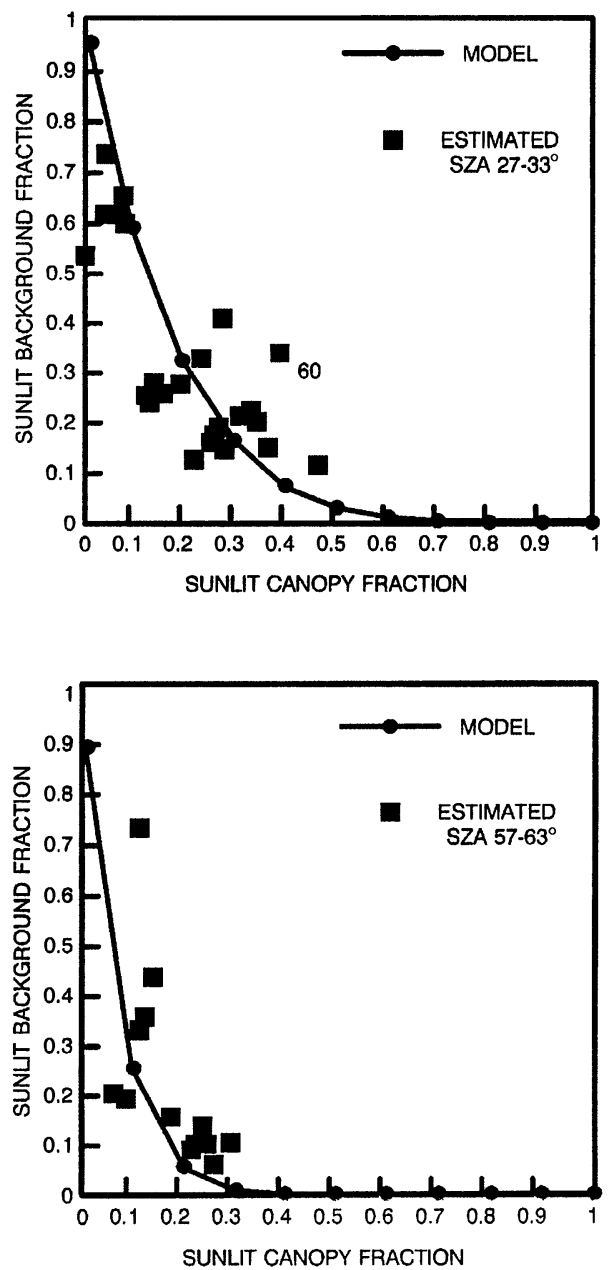

sunlit canopy. At a $30^{\circ} \mathrm{SZA}$, the critical density is $\approx 40 \%$, while at $60^{\circ}$ it is $\approx 25 \%$.

In Fig. 5 is plotted mixture model extracted sunlit background versus sunlit canopy fraction, corroborating the cylindrical canopy model predictions that sunlit background fraction will decrease as sunlit vegetation fraction increases and progressively obscures the sunlit background with sunlit canopy and shadow. Once again, as the solar zenith angle increases, the obscuration of the sunlit background becomes more rapid as a result of enhanced shadowing.

\section{Relationship of the end member fractions to stand biophysical parameters}

The work in the previous section demonstrates that remote sensing data can be used to extract shadow fraction, sunlit canopy fraction, and sunlit background fraction. However, these parameters, in and of themselves, are not used directly in earth system process models.

Our results show that these end member fractions are strongly related to stand structural parameters that are of interest to such process models. Note in Fig. 6 that the shadow fraction of the black spruce stands as estimated from the helicopter MMR data is strongly

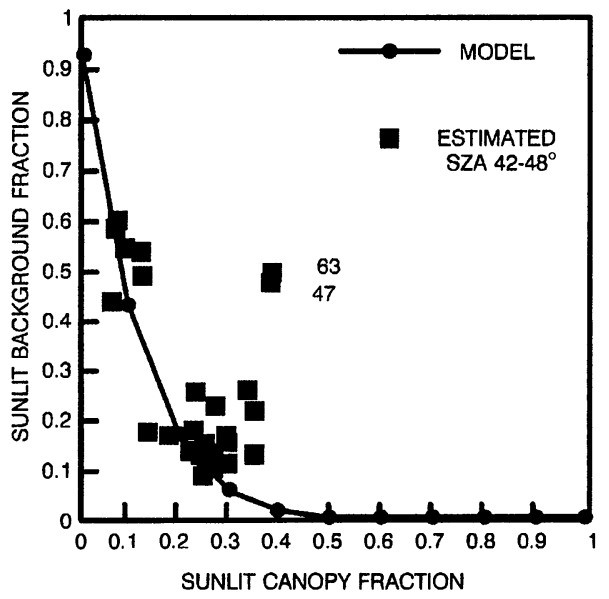

FIG. 5. Fraction of sunlit background versus fraction of sunlit vegetation as estimated by the mixture decomposition algorithm when applied to the helicopter-mounted MMR reflectance measurements over black spruce stands in the Superior National Forest. MMR data were acquired for solar zenith angle ranges of $27^{\circ}-33^{\circ}, 42^{\circ}-48^{\circ}$, and $57^{\circ}-63^{\circ}$. Plotted for comparison are the cylindrical canopy model predicted values of the end member fractions. related to biomass density. Fig. 6a-c show the dependence of the relationship on SZA. There is not a significant difference in the relationship between SZA ranges of $27^{\circ}-33^{\circ}$ and that of $42^{\circ}-48^{\circ}$, as shown in Fig. $6 \mathrm{~d}$, which combines the data from these ranges. As shown in Fig. 7, sunlit background fraction is also strongly related to these biophysical parameters. The relationship however, is strongest below the critical density, corresponding to a biomass density of $\approx 6 \mathrm{~kg}$ / $\mathrm{m}^{2}$.

At the same time biomass density measurements were acquired for the black spruce stands, other biometric measurements were taken as shown in Table 3. In Fig. 8, these parameters are also significantly correlated to biomass density, so that the mixture fractions can provide useful estimates of these additional parameters. All of these parameters: leaf area index (LAI), diameter at breast height (dbh), aboveground net primary productivity (NPP), and basal fraction are useful inputs to ecosystem process models.

Table 6 shows the standard error of regression (for a linear fit) for the various end member fractions as estimators of biomass density, LAI, dbh, and NPP for SZAs of $30^{\circ}, 45^{\circ}$, and $60^{\circ}$. Overall, shadow fraction 

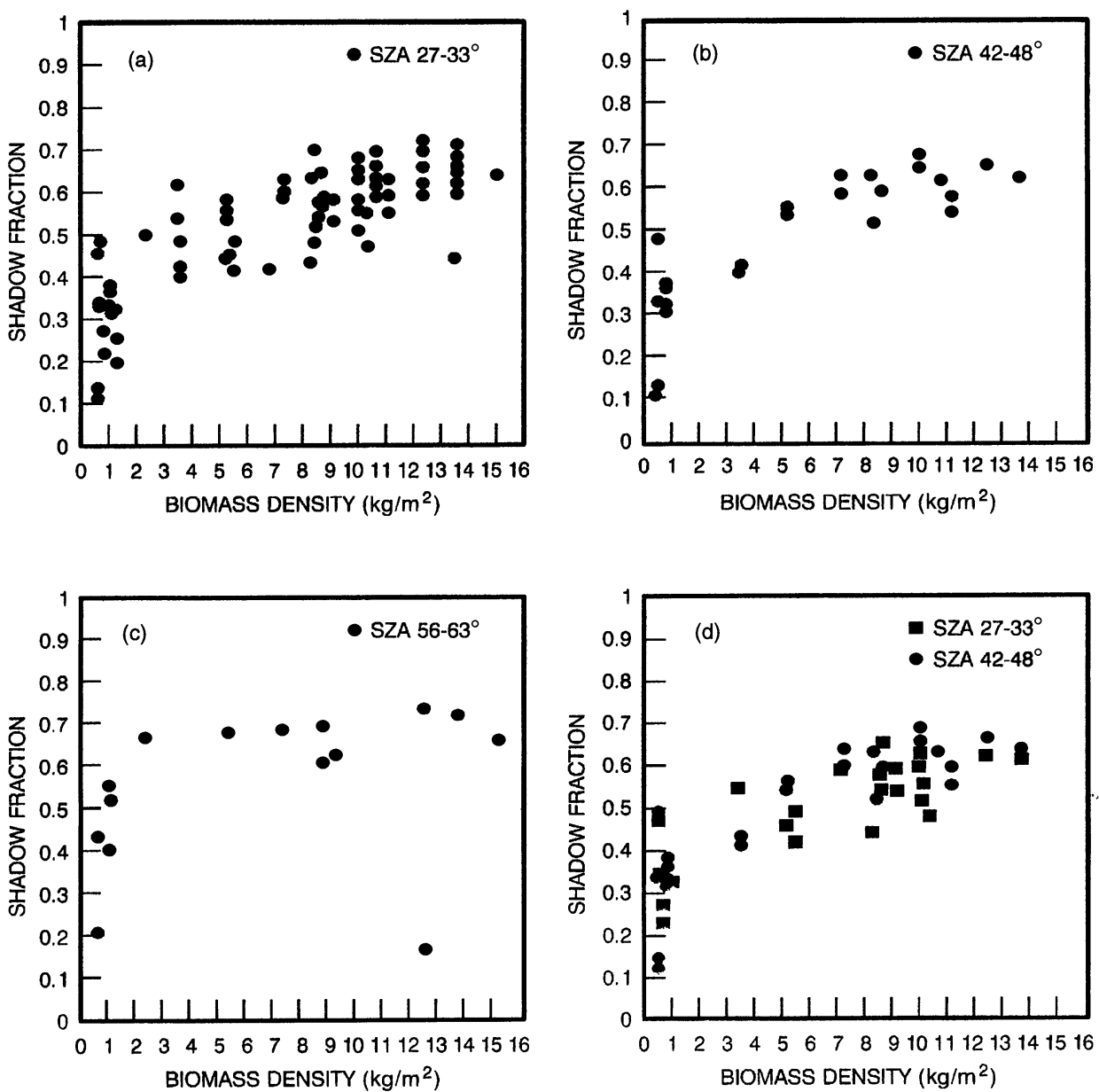

FIG. 6. Relationship between shadow fraction and biomass density for Superior National Forest black spruce stands. MMR data were acquired for solar zenith angle ranges of (a) $27^{\circ}-33^{\circ}$, (b) $42^{\circ}-48^{\circ}$, (c) $57^{\circ}-63^{\circ}$, and (d) combined data from (a) and (b).

appears to be the best estimator of these variables, showing the largest $r^{2}$ and the smallest standard errors with all the biophysical variables; a SZA of $30^{\circ}$ is "best" in the same sense. These results show that biomass can be estimated with a standard error of $\approx 2 \mathrm{~kg}$ / $\mathrm{m}^{2}$, LAI with a standard error of 0.58 , dbh with a standard error of $\approx 2 \mathrm{~cm}$, and aboveground NPP with a standard error of $0.07 \mathrm{~kg} \cdot \mathrm{m}^{-2} \cdot \mathrm{yr}^{-1}$.

Note that in Fig. 9, the fraction of sunlit canopy also bears a functional relationship to biomass density, albeit much weaker than sunlit background or shadow fraction to the point of being not useful. This is reflected in Table 6, where the $r^{2}$ with the biophysical parameters is small. This can be explained in part by the fact that the range in sunlit canopy fraction is not as great as that of shadow or sunlit background. As canopy density becomes large, self-shadowing by crowns will define a limiting value for sunlit canopy fraction, seen in Fig. 9 at $\approx 0.3-0.4$. This limit does not show up in our cylindrical canopy model, but it is a reality with conical tree crowns.
While the correlations demonstrated here have been purely empirical ones, they suggest the existence of a less heuristic connection between the end member fractions and biophysical characteristics through the relationship of canopy biomass to canopy spatial dimensions and thus shadowing. We will address these possibilities more fully in the Discussion.

\section{Why NDVI fails as a surrogate for stand biophysical parameters}

A large number of regional and global studies have relied on NDVI to construct vegetation density maps. Our results show, in Fig. 10a, that NDVI is not a reliable predictor of biophysical parameters for the dominant coniferous species of the boreal forest. Fig. 10b shows why! NDVI is linear in sunlit canopy fraction $\left(r^{2}=0.96\right)$ and, as we have already seen, sunlit canopy fraction is not strongly related to biomass density. However, NDVI is still a useful index for two calculations:

1) It can be used to estimate sunlit canopy fraction, 

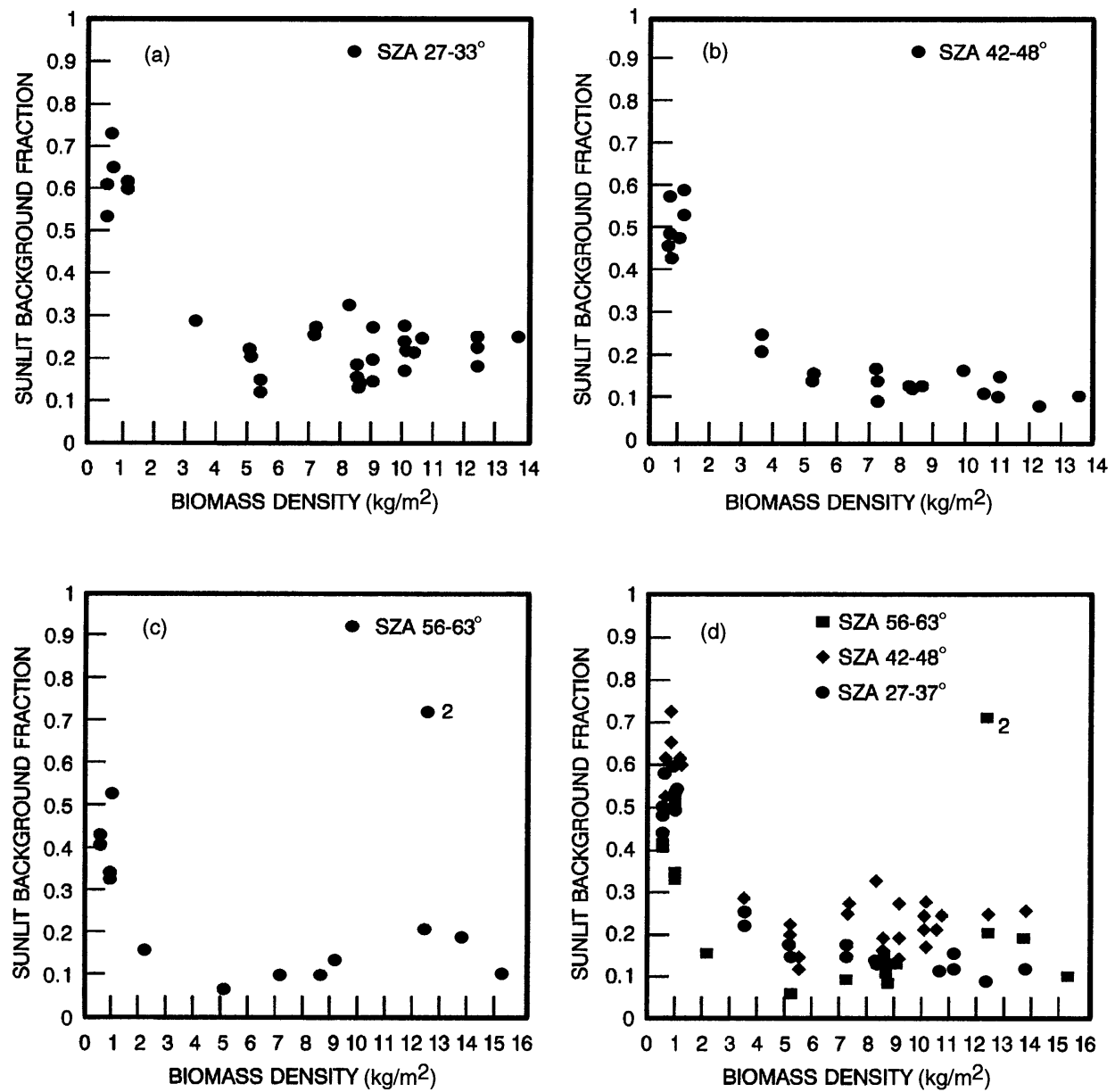

FIG. 7. Relationship between sunlit background fraction and biomass density for Superior National Forest black spruce stands. MMR data were acquired for solar zenith angle ranges of (a) $27^{\circ}-33^{\circ}$, (b) $42^{\circ}-48^{\circ}$, (c) $57^{\circ}-63^{\circ}$, and (d) combined data from (a) and (b).

which is in turn directly related to the canopy intercepted fraction of photosynthetically active radiation $\left(F_{\text {ipar }}\right)$. From $F_{\text {ipar }}$ we can compute $F_{\text {par }}$, the canopy fraction of absorbed photosynthetically active radiation, given the reflectance and transmission of the canopy and the background reflectance (from either measurement or radiative transfer models).

2) Using NDVI to obtain an estimate of sunlit canopy fraction can yield estimates of the sunlit canopy reflectance, the shadow fraction $S$, and the sunlit background fraction, from simultaneous solutions to Eqs. 1 and 4 , given values for shadow reflectance and sunlit background reflectance.

\section{Discussion}

In the proposal of a new procedure that relies on spectral information similar or identical to that used in earlier attempts to estimate vegetation parameters, it is reasonable to ask how the new approach differs from the old. We have attempted to do that to some degree in the Introduction, and will attempt to further that aim in this section.
In this discussion we will address five fundamental questions: (1) What are the effects of varying illumination angle on our results? (2) What is the effect of atmospheric transmission and scattering loss on these results? (3) Why is NDVI so highly correlated to sunlit canopy fraction? (4) How does the mixture decomposition approach to estimating biophysical parameters relate to approaches relying on vegetation index? (5) How does the allometry used to establish relationships among individual tree morphology and biophysical characteristics influence the observed empirical correlations with end member fractions?

\section{Illumination angle effects on red vs. NIR reflectance}

As we saw from the theoretical and measured values of end member fractions in Figs. 2, 4, and 6, the SZA affects the relationships between these fractions. This in turn propagates into the reflectance scatter plot in Fig. 3. The structure of this scatter plot can be better appreciated in Fig. 11, where we have used Eq. 1 to compute reflectance as it varies with $C . S$, in turn, is computed from Eqs. 2 and 3 for a range of SZA. Each 

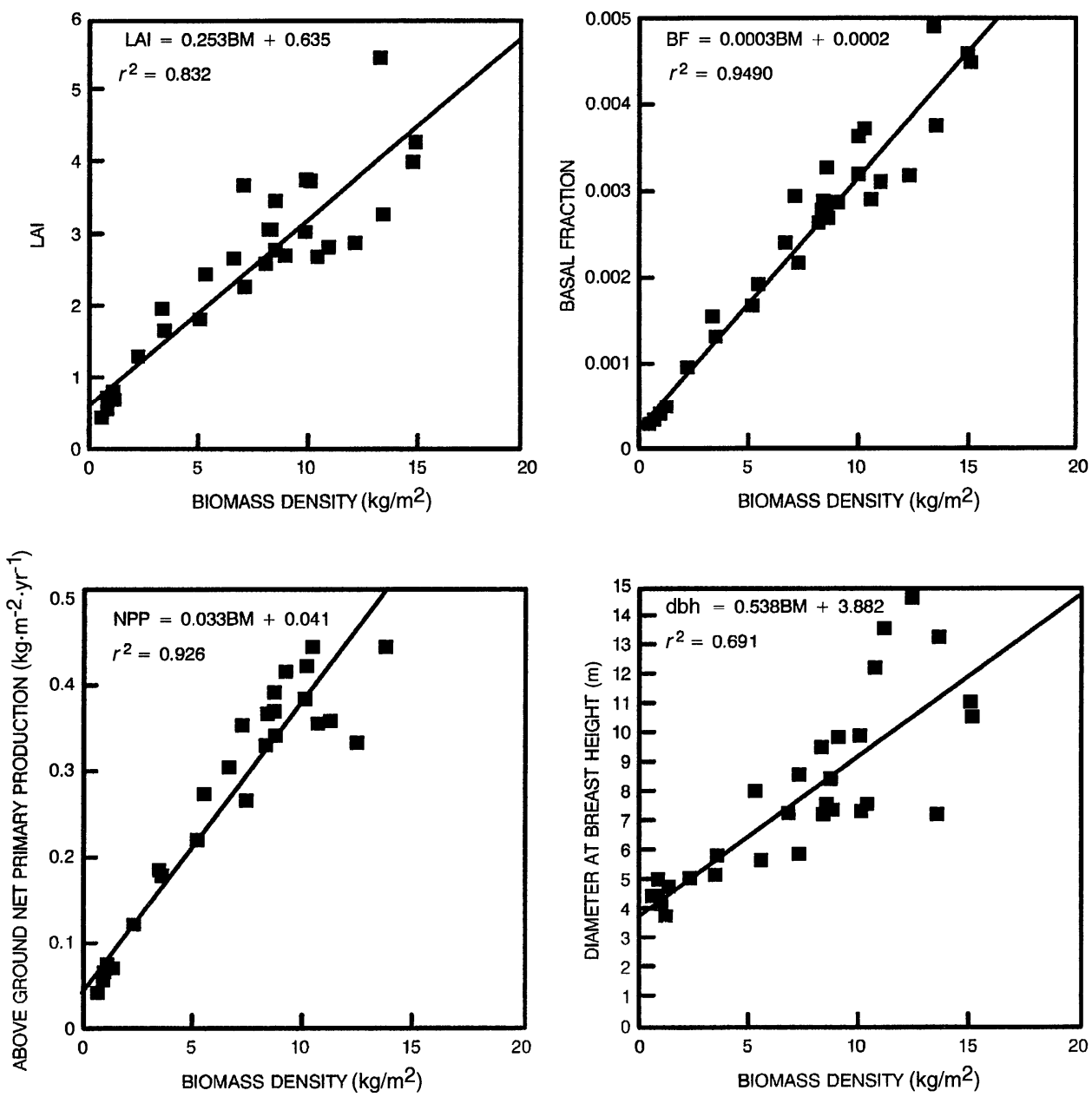

FIG. 8. Relationship between biomass density, LAI, basal fraction, dbh, and NPP.

point on the plot represents equal increments of sunlit canopy fraction, varying from $0-1$ in 0.025 increments. The outer envelope of the red-NIR scatter gram is determined by larger SZAs. At the other extreme, where the illumination is from nadir (not possible in practice at higher latitudes), the variation in red-near infrared reflectance will be along a line joining the background and sunlit canopy reflectance values. All other red-NIR values will fall within the triangle formed by the maximum solar illumination angle, which has vertices at

TABLE 6. Standard error of regression and correlation coefficients among biomass density, LAI, dbh, and NPP (columns) and shadow fraction and sunlit background fraction at $30^{\circ}, 45^{\circ}$, and $60^{\circ} \mathrm{SZA}$ (rows).

\begin{tabular}{|c|c|c|c|c|c|c|c|c|}
\hline & \multicolumn{2}{|c|}{ Biomass } & \multicolumn{2}{|c|}{ LAI } & \multicolumn{2}{|c|}{$\mathrm{dbh}$} & \multicolumn{2}{|c|}{ NPP } \\
\hline & $r^{2}$ & $\mathrm{SE}$ & $r^{2}$ & $\mathrm{SE}$ & $r^{2}$ & $\mathrm{SE}$ & $r^{2}$ & SE \\
\hline \multicolumn{9}{|l|}{$\mathrm{SZA}=30^{\circ}$} \\
\hline $\begin{array}{l}\text { Canopy } \\
\text { Background } \\
\text { Shadow }\end{array}$ & $\begin{array}{l}0.21 \\
0.60 \\
0.76\end{array}$ & $\begin{array}{l}3.65 \\
2.60 \\
2.00\end{array}$ & $\begin{array}{l}0.42 \\
0.74 \\
0.72\end{array}$ & $\begin{array}{l}0.84 \\
0.56 \\
0.58\end{array}$ & $\begin{array}{l}0.04 \\
0.31 \\
0.54\end{array}$ & $\begin{array}{l}2.86 \\
2.43 \\
1.97\end{array}$ & $\begin{array}{l}0.39 \\
0.73 \\
0.74\end{array}$ & $\begin{array}{l}0.11 \\
0.07 \\
0.07\end{array}$ \\
\hline \multicolumn{9}{|l|}{$\mathrm{SZA}=45^{\circ}$} \\
\hline $\begin{array}{l}\text { Canopy } \\
\text { Background } \\
\text { Shadow }\end{array}$ & $\begin{array}{l}0.09 \\
0.77 \\
0.71\end{array}$ & $\begin{array}{l}4.25 \\
2.11 \\
2.41\end{array}$ & $\begin{array}{l}0.10 \\
0.79 \\
0.70\end{array}$ & $\begin{array}{l}0.07 \\
0.51 \\
0.62\end{array}$ & $\begin{array}{l}0.08 \\
0.62 \\
0.54\end{array}$ & $\begin{array}{l}3.34 \\
2.14 \\
2.35\end{array}$ & $\begin{array}{l}0.10 \\
0.82 \\
0.73\end{array}$ & $\begin{array}{l}0.14 \\
0.06 \\
0.07\end{array}$ \\
\hline \multicolumn{9}{|l|}{$\mathrm{SZA}=60^{\circ}$} \\
\hline $\begin{array}{l}\text { Canopy } \\
\text { Background } \\
\text { Shadow }\end{array}$ & $\begin{array}{l}0.00 \\
0.14 \\
0.25\end{array}$ & $\begin{array}{l}4.80 \\
4.46 \\
4.16\end{array}$ & $\begin{array}{l}0.20 \\
0.62 \\
0.49\end{array}$ & $\begin{array}{l}1.23 \\
0.85 \\
0.99\end{array}$ & $\begin{array}{l}0.04 \\
0.12 \\
0.40\end{array}$ & $\begin{array}{l}3.47 \\
3.31 \\
2.74\end{array}$ & $\begin{array}{l}0.17 \\
0.54 \\
0.43\end{array}$ & $\begin{array}{l}0.16 \\
0.12 \\
0.13\end{array}$ \\
\hline
\end{tabular}



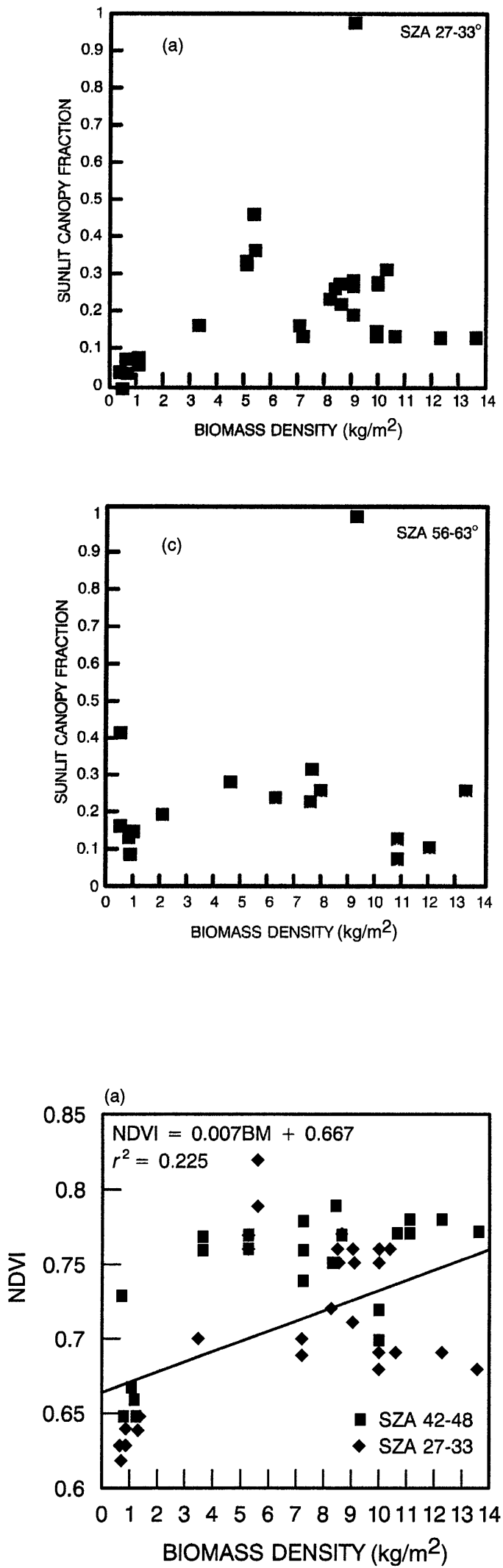

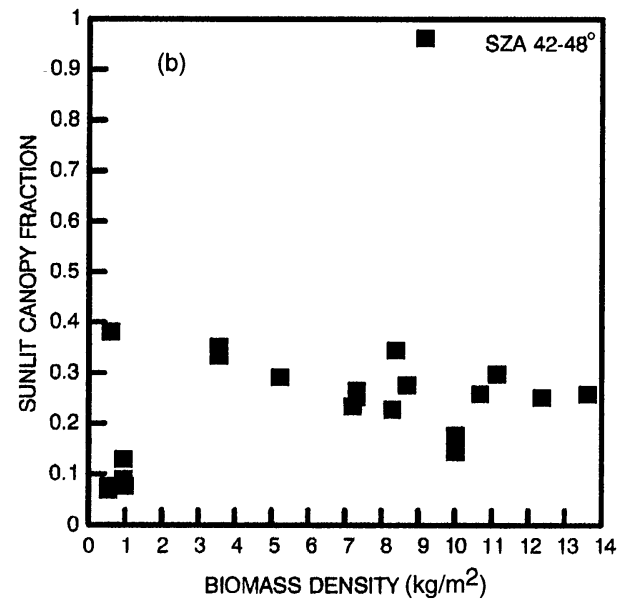

FIG. 9. Relationship between sunlit canopy fraction and biomass density for Superior National Forest black spruce stands. MMR data were acquired for solar zenith angle ranges of (a) $27^{\circ}-33^{\circ}$, (b) $42^{\circ}-48^{\circ}$, and (c) $57^{\circ}-$ $63^{\circ}$.

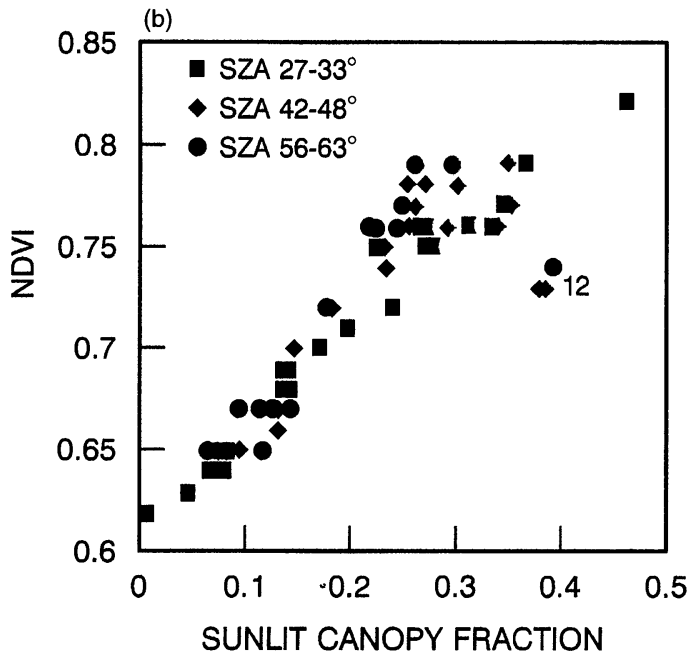

FIG. 10. (a) NDVI versus biomass density. (b) NDVI versus fraction of sunlit crown. Both figures for black spruce stands and SZAs of $27^{\circ}-48^{\circ}$. 


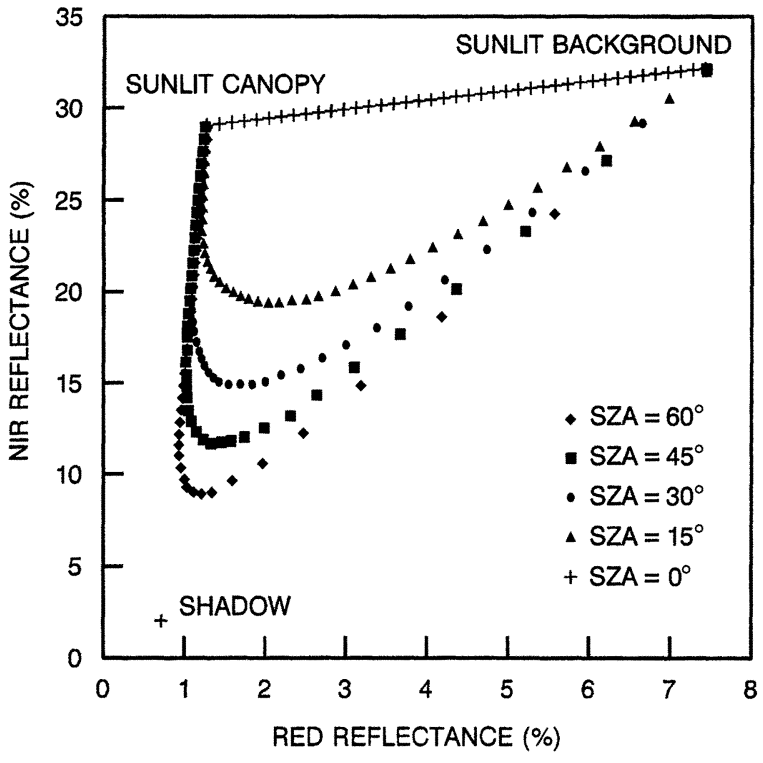

FIG. 11. Cylinder model predictions of red versus nearinfrared reflectance for SZA of $0^{\circ}, 15^{\circ}, 30^{\circ}, 45^{\circ}$, and $60^{\circ}$. Each point, starting from sunlit background, represents an equal increment of sunlit canopy fraction of 0.025 increasing from 0 ("sunlit background" point on the graph) to 1 ("sunlit canopy" point on the graph). The graph illustrates the effects of solar illumination angle on observed reflectance. End member reflectances are as derived in Methods.

the critical density point on the vertex, and the sunlit sphagnum and sunlit canopy reflectance values. Thus the triangle of measured reflectance values in Figs. 1 and 3 is explained by the fact that the reflectance measurements came from an assortment of stands of varying density with acquired at SZAs varying from $27^{\circ}-$ $75^{\circ}$. It is this structure that gives rise to the ability to relate shadow and sunlit canopy fraction to biophysical parameters.

\section{Graphical interpretation of mixture fractions}

While Eqs. 5 and 6 rigorously describe the mixture decomposition algorithm, a graphical representation of their solutions adds intuitive understanding and insight into how the mixture decomposition approach will be affected by variability in the end member signatures and other error sources such as atmospheric attenuation and scattering. Fig. 12 provides such a representation, since the lines inside the end member simplex (triangle whose vertices are the end member locations in reflectance space) represent lines of constant end member fractions as the SZA varies. The proof of this is derived in the Appendix. The end member values are the ones used in our black spruce study. Plotted is a hypothetical data point represented by the open circle. For such a data point, $S$ would equal 0.6 , and $B$ and $C$ would each equal 0.2 .

Thus the graphical mixture decomposition algorithm for computing the proportions for three mixtures con- tained in a pixel from its reflectance in two bands is quite simple:

1) Draw a simplex containing the end member reflectance values as vertices.

2) Draw three lines through the pixel location within the simplex such that each line is parallel to a side of the simplex. The reflectance line of constant proportion for an end member is parallel to the simplex side opposite the end member vertex.

3) The end member proportion for the pixel is the ratio of: (a) the perpendicular distance from the pixel to the simplex side opposite the end member, and (b) the perpendicular distance from the side opposite the end member vertex to a parallel line passing through the end member vertex.

\section{NDVI and sunlit canopy fraction}

Fig. 10b shows a remarkable correlation between NDVI and sunlit canopy fraction. Fig. 13, an overlay of lines of constant NDVI on Fig. 11, shows why. When NDVI is constant,

$$
\text { NDVI }=\left(\rho_{t 1}-\rho_{t 2}\right) /\left(\rho_{t 1}+\rho_{t 2}\right)=K,
$$

which can be solved for $\rho_{t 2}$ in terms of $\rho_{t 1}$ :

$$
\rho_{t 1}=\rho_{t 2}(1+K) /(1-K)
$$

Fig. 13 shows the constant lines of NDVI $=0$, NDVI $=$ sunlit background $=0.63, \mathrm{NDVI}=0.71, \mathrm{NDVI}=$ maximum shadow $=0.79$, and $\mathrm{NDVI}=$ sunlit canopy

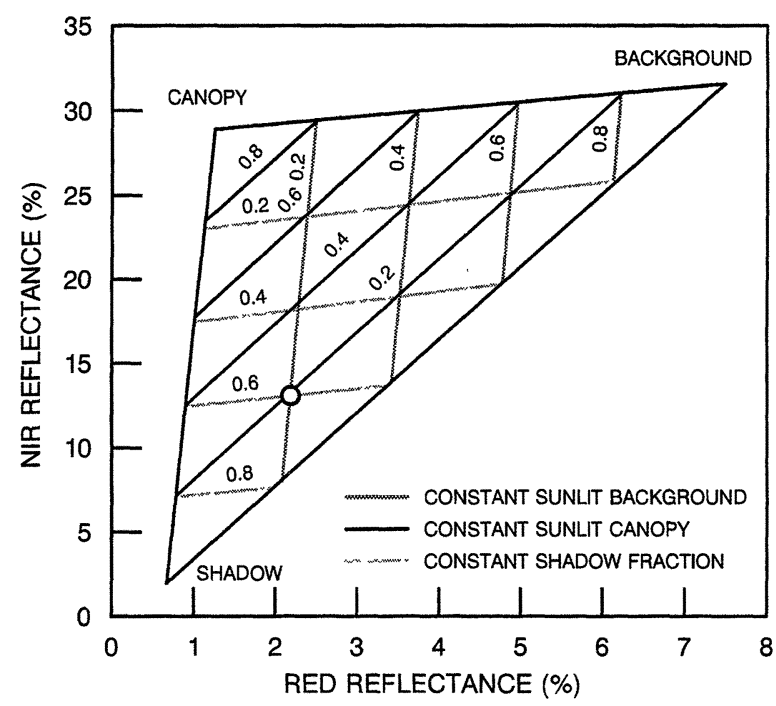

FIG. 12. Graphical approach to inferring mixture proportions given red and NIR reflectance. Each side of the triangular simplex is a solution for an end member proportion of zero, for the end member opposite that side. Lines within the end member simplex are solutions to Eqs. 1 and 4 assuming constant end member proportions (see Appendix for derivation). Annotated values on lines represent values of constant end members. Open circle represents value of red-NIR reflectance. End member proportions for that pixel given by values for lines intersecting that reflectance value; $S=0.6$, and $B=0.2$, and $C=0.2$. 


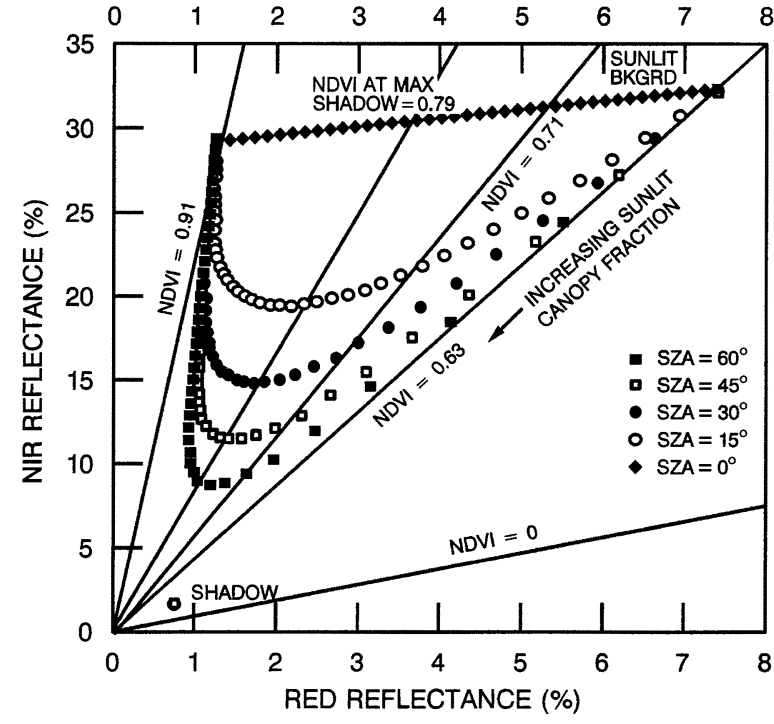

FIG. 13. Constant lines of NDVI for NDVI $=0$, NDVI $=$ canopy background $=0.63, \mathrm{NDVI}=0.71, \mathrm{NDVI}=\max -$ imum shadow $=0.79$, and NDVI $=$ sunlit canopy $=0.91$. As canopy fraction increases, NDVI increases almost linearly from its sunlit background value of 0.63 . NDVI increases at the same rate for all SZA, rendering the relationship between NDVI and sunlit canopy fraction independent of SZA.

$=0.91$. Since the plotted points in Fig. 13 are calculated for constant increments of sunlit canopy fraction, we see that as canopy fraction increases, NDVI increases almost linearly from its sunlit background value of 0.63 , and at similar rates for all SZA. This independence of the NDVI-sunlit canopy fraction relationship to SZA can also be seen in Fig. 10b. In Fig. 12, note that lines of constant sunlit canopy fraction in the simplex are nearly parallel to lines of constant NDVI for low sunlit canopy fraction, but become increasingly divergent at high sunlit canopy fraction. Note in Fig. $10 \mathrm{~b}$ that we do see a saturation of NDVI sensitivity at an NDVI of $\approx 0.79$, the NDVI at maximum shadow. As can also be appreciated from Figs. 12 and 13, the sensitivity of NDVI to sunlit canopy fraction depends on the difference between sunlit background and sunlit canopy reflectance, particularly in the visible part of the spectrum. As the sunlit canopy reflectance approaches the sunlit background reflectance, the rate of change of NDVI with sunlit canopy decreases. When the NDVI of the sunlit canopy is exactly that of the NDVI of maximum shadow, NDVI actually begins to decrease with sunlit canopy fractions beyond the critical density. Note that this value is almost independent of SZA and equal to $\approx 0.79$. For vegetated communities where the NDVI of the sunlit canopy is equal to that of the sunlit background, NDVI will be totally insensitive to sunlit canopy fraction.

\section{Atmospheric effects on the mixture decomposition algorithm}

A problem of concern in any remote sensing application, particularly in regional to global ones, is the effect of atmospheric conditions on the surface reflectance. In the mixture modeling application, variations in atmospheric conditions from one date to the next, or in one region to the next, would modify the values of the end member reflectances with the potential for creating error in the estimates of end member mixing proportions within a pixel. In this section, we will examine such effects.

Over a region in which the atmosphere can be considered horizontally homogeneous (aerosols, water vapor, and molecular scattering and absorption), the atmospherically transformed reflectance in the ith band and can be expressed approximately as

$$
\rho_{t i}=S_{i}+T_{i} \rho_{\iota}
$$

where $S_{l}$ is the path scattering contribution and $T_{1}$ represents path transmission. Fig. 14 shows the effect on the simplex of Fig. 12 and its vertices when a transformation of the type shown in Eq. 9 is applied. In this example, the magnitude of path scattering is assumed to be $2 \%$ reflectance for red and $0.1 \%$ for NIR; transmission is assumed to be 0.8 for red and 0.95 for NIR. Note that the mixture proportions remain invariant to linear transformations since the end member reflectances and the mixture pixel reflectances are affected identically. Thus, if a procedure can be developed to select end members based on their relative positions in spectral space (opposed to absolute reflectance values),

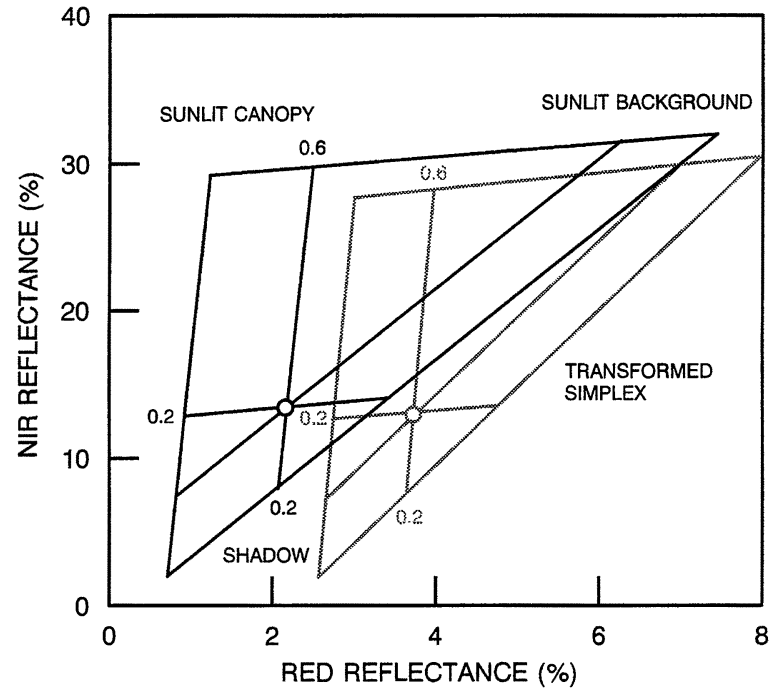

FIG. 14. Effect of atmospheric scattering and absorption on the end member simplex. The simplex with solid lines is the black spruce canopy-sphagnum background used in this study. The simplex formed with dotted lines would result from the same reflectance data being acquired under atmospheric conditions corresponding to a path scattering of $2 \%$ reflectance for red and $0.1 \%$ for NIR with an atmospheric transmission of 0.8 for red and 0.95 for NIR. Note that the end member proportions of the open circle are invariant under this transformation; this results from the fact that the end member and pixel reflectance undergo identical, linear transformations. 


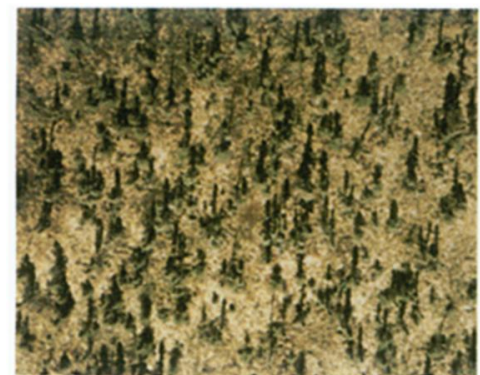

Shadow $=0.19$

Sunlit Canopy $=0.00$

Sunlit Bkg $=0.81$

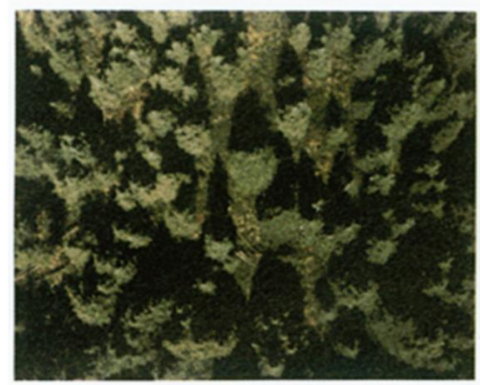

Shadow $=0.42$

Sunlit Canopy $=0.46$

Sunlit Bkg $=0.12$

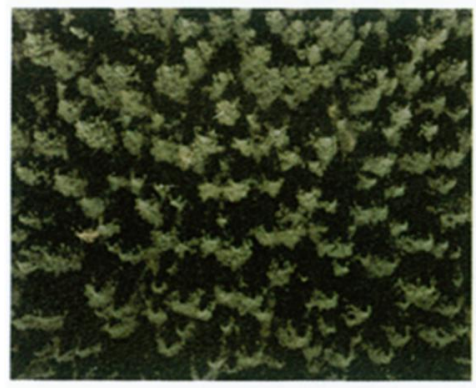

Shadow $=0.54$

Sunlit Canopy $=0.27$

Sunlit Bkg $=0.19$

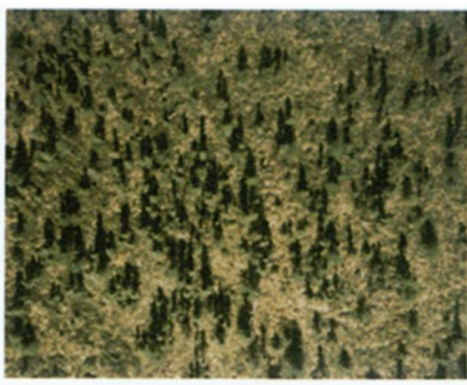

Shadow $=0.27$

Sunlit Canopy $=0.08$

Sunlit Bkg $=0.65$

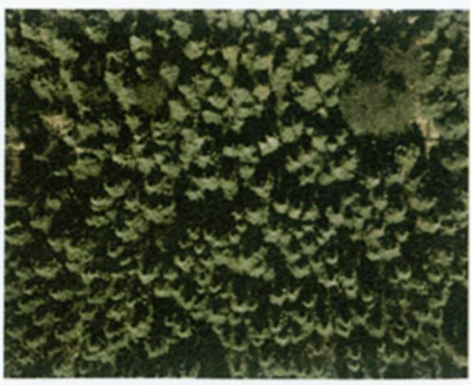

Shadow $=0.48$

Sunlit Canopy $=0.31$

Sunlit $\mathrm{Bkg}=0.21$

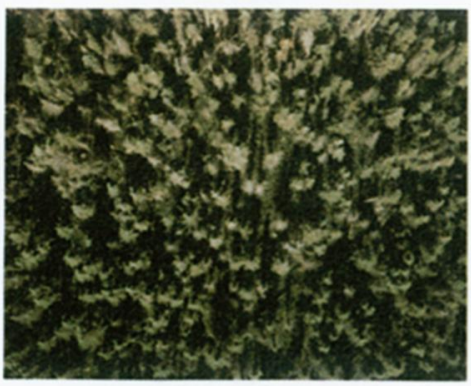

Shadow $=0.54$

Sunlit Canopy $=0.17$

Sunlit $\mathrm{Bkg}=0.29$

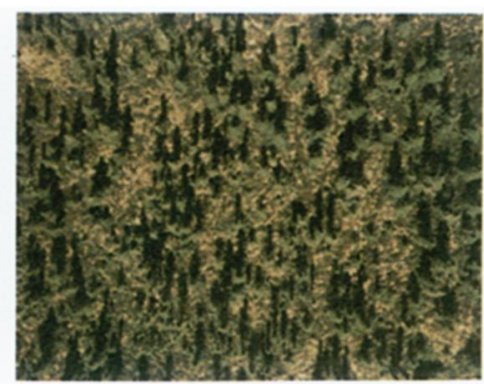

Shadow $=0.32$

Sunlit Canopy $=0.08$

Sunlit $\mathrm{Bkg}=0.60$

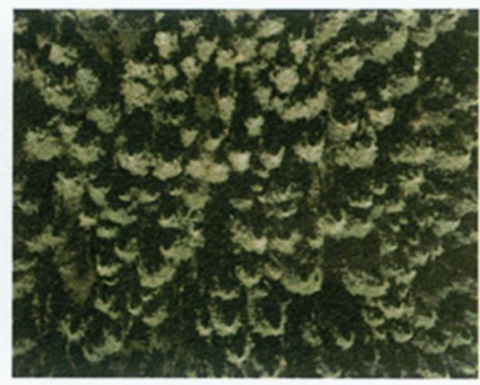

Shadow $=0.53$

Sunlit Canopy $=0.28$

Sunlit Bkg $=0.19$

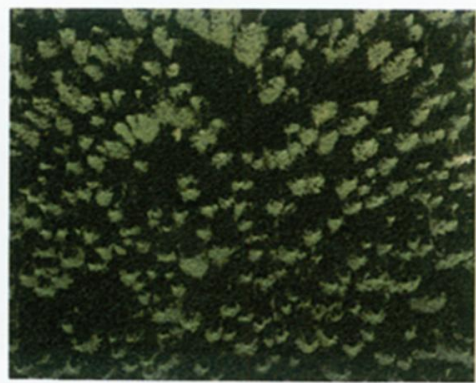

Shadow $=0.61$

Sunlit Canopy $=0.13$

Sunlit Bkg $=0.26$

FIG. 15. Vertical helicopter photography of Superior National Forest black spruce stands with end member fractions as estimated from the mixture decomposition algorithm.

the mixture decomposition algorithm will be invariant to atmospheric differences between scenes, eliminating the need for atmospherically corrected data. As we will discuss in the Discussion: Developing an end memberbased regional or global procedure, it may be possible to select end members based on their relative positions or satellite digital count values, and thus avoid the need for both calibration and atmospheric correction.

\section{Errors arising from noise in end member reflectances}

From the positions of sites 12 and 103 in Fig. 3, one would estimate that they are high biomass density black spruce sites. In fact, if we look at Table 3, we see that both sites are relatively low biomass density sites. Both sites have large proportions of Iarch (Larix laricina) in comparison to the other, nearly pure black spruce sites. Larch is a deciduous canopy, and the data from the "outlier" sites in Fig. 3 were acquired in July and August 1983 and June 1984, when larch is much more reflective than black spruce, particularly in the NIR. Thus, at the same biomass density, larch is more reflective than black spruce. Without adjusting the sunlit canopy end member reflectance for this difference, the use of pure black spruce end members in the mixture decomposition of stands containing larch creates substantial error in the estimate of end member proportions and corresponding errors in biophysical parameters 

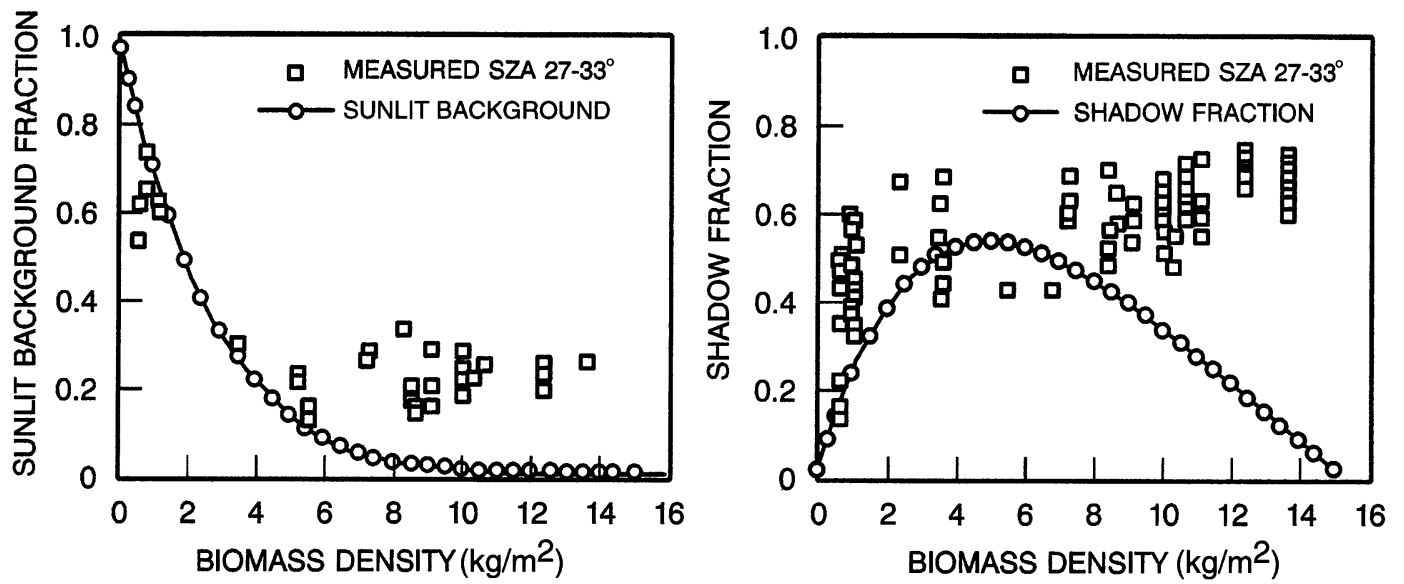

FIG. 16. Modeled values of sunlit background and shadow fraction as a function of biomass density. Model is as shown in Eqs. 11 and 12.

such as biomass density. The effects of this can be seen in Fig. 4b for site 12, and in Fig. 5b for site 63; both sites contain $12 \%$ larch. Thus errors in either sunlit canopy or sunlit end member reflectances can create large errors in the estimation of background end member proportions because of the relatively small difference between sunlit background and sunlit canopy reflectance. On the other hand, proportion estimation errors are relatively insensitive to errors in the shadow reflectance because it is quite different from either sunlit canopy or sunlit background. To mitigate the operational effects of these errors, one approach would be to stratify the region into community composition classes with significantly different end member reflectances (e.g., conifer, deciduous, etc.) using image classification approaches, and apply the mixture decomposition algorithms using different end members within the different strata.

\section{End member fractions and biophysical characteristics}

Fig. 15 shows helicopter-acquired vertical color photography from 9 of the 31 black spruce stands used in this study. What can be seen in this array of photographs is visual proof of the empirical correlations observed earlier between the various end member fractions and biomass density. Shown beneath each of the photographs are the estimates of mixture fractions derived from the red-NIR reflectance of these stands. As the stands get denser and biomass density increases, sunlit canopy and shadows increase, while sunlit background decreases. The lowest density stands are nutrient and oxygen-deprived bogs with very low aboveground net primary productivity. The nine stands span a gradient of productivity, increasing as soils become better drained and thus more supportive of growth.

Using the cylinder model employed here to represent tree canopy geometry, we can partially quantify the relationship between shadow, sunlit background, and biomass density by assuming that biomass density is proportional to $C$. With this assumption we may define a new variable, biomass density fraction (BMD $f$ ), as the ratio of the biomass density BMD at a sunlit canopy fraction of $C$ to the biomass density at complete canopy cover, i.e.,

$$
\mathrm{BMD} f=\mathrm{BMD} / \mathrm{BMD}_{\max }
$$

where $\mathrm{BMD}=\mathrm{BMD}_{\max }$ when $C=1$. Then the shadow fraction as given in Eq. 2 could be written as:

$$
S=1-\operatorname{BMD} f-(1-\operatorname{BMD} f)^{(\eta+1)},
$$

and, from Eqs. 4 and 2, sunlit background fraction is proportional to

$$
B=(1-\operatorname{BMD} f)^{(\eta+1)} .
$$

A crude estimate of $\mathrm{BMD}_{\max }$ is simply the largest value of BMD observed on any of the SNF sites, which was $15.04 \mathrm{~kg} / \mathrm{m}^{2}$. Using this value, we compute and plot Eqs. 11 and 12, overlayed in Fig. 16 with the empirical values of end member fractions versus biomass density. Two problems with the cylinder model are immediately obvious. In Fig. 16a, we see that for high values of biomass density, Eq. 11 does not correctly predict the empirically obtained values of shadow fraction versus biomass. This is a result of the fact that in the simple cylinder model, shadow fraction goes to zero as sunlit canopy approaches unity. In the densest of real SNF black spruce canopies of Fig. 15, the sunlit canopy fraction cannot be unity, because the canopy is rough and will produce shadows.

While this simple analysis is approximate, it does point the way toward a more sophisticated analysis to relate shadow and sunlit background fraction to canopy biophysical parameters, using more realistic models between canopy geometry and biophysical parameters.

That sunlit canopy fraction does not correlate with 

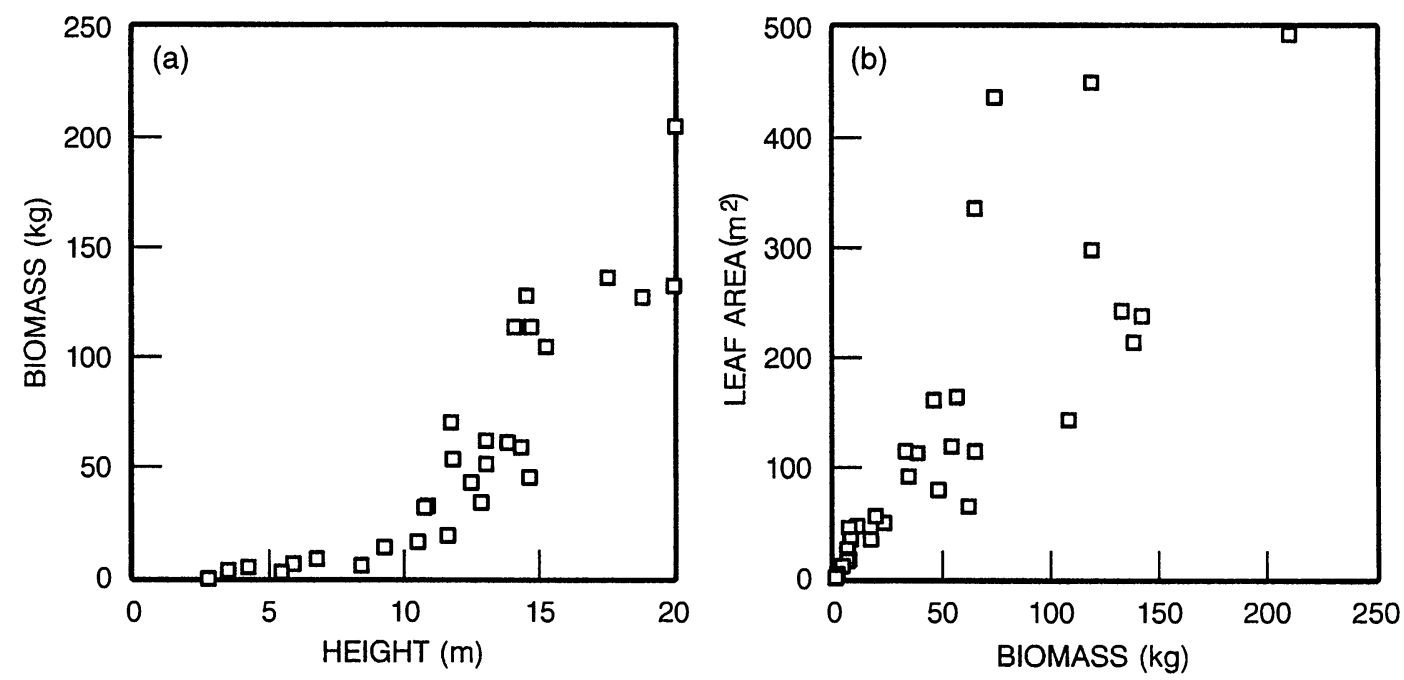

FIG. 17. (a) Individual tree biomass versus tree height and (b) individual tree leaf area versus biomass as determined from sacrificed black spruce trees in the Superior National Forest study.

the biophysical parameters (Fig. 9) is somewhat of a curiosity since the cylinder model predicts that it should. Note that in Fig. 9, at all three sun angles, sunlit canopy fraction does increase initially with biomass density until the critical density is reached, then it begins to decrease (although these correlations are very noisy). That the range of sunlit canopy is much smaller than that of shadow or sunlit background explains part of the lack of correlation. However, it may also be the case that non-linear effects, such as the dependence of sunlit canopy or shadow reflectance with crown depth, or morphological differences with tree size, may lead to this behavior. This is a question requiring further investigation.

\section{Correlation among the biophysical parameters}

A brief discussion is in order regarding the remarkable correlation among the many stand-level biophysical characteristics observed in Fig. 8. None of the stand-level characteristics shown in Fig. 8 are measured directly; rather, allometric relations are used to compute these characteristics, primarily from $\mathrm{dbh}$ at the individual tree level. Tree-level values of biomass and leaf area are estimated from values of $\mathrm{dbh}$ for the individual trees, then aggregated to estimate average biomass density and leaf area index at the plot and stand level. Fig. 17 shows why the stand-level biophysical variables shown in Fig. 8 are all correlated with each other, and thus with end member fractions. These treelevel biophysical parameters were measured from 30 sacrificed black spruce trees in the SNF. Because tree height is so highly correlated to biomass and leaf area, and because the height to width ratios for black spruce canopies in the SNF are relatively constant, a high correlation between canopy volume, canopy biomass, and canopy leaf area is introduced. As we have seen from the cylindrical canopy model, canopy volume is in turn related to sunlit background and shadow fraction.

\section{Developing an end member-based regional or global procedure}

As we have seen from our evaluation, the following conditions are essential for the implementation of mixture decomposition to estimate scene proportions: (1) accurate estimates of at least the relative position of the end members in spectral space, (2) the differences in the end member reflectances must be large in comparison to the reflectance variability in the scene, and (3) if absolute values are to be used for end member reflectance, satellite images must be calibrated and atmospherically corrected.

Sunlit canopy and background end member reflectances vary with species composition and background; thus, mixture decomposition must be applied to areas consisting of a single community type. The first requirement of a regional or global mixture decomposition procedure, therefore, is a reliable community and composition classification. In our particular case, we would need a reliable map identifying the black spruce stands. Fortunately, there has been a large amount of research focusing on image classification and this task is quite achievable.

Even with species composition given, satellite data must be corrected for calibration drift and atmospheric effects if preset values of sunlit canopy, shadow, and sunlit background reflectances are to be used in mixture decomposition. When atmospheric optical depth or calibration data are not available to correct the satellite images, relative digital count values for end members must be determined directly from, and applied to, each image. One possible approach to this relative method is as follows. If treeless fens can be located from classification or photo interpretation, the uncorrected dig- 
ital count values for those areas could serve as end member values for the sphagnum background; treeless bogs are often covered with sphagnum as are the treed fens and bogs. Shadow digital count values could be estimated from clear lakes if available. This leaves sunlit canopy reflectance as the only undetermined value. However, assuming the cylindrical canopy model holds, Eqs. 1, 2, and 4 may be combined to yield

$$
\begin{aligned}
\rho_{t t}= & C \rho_{c t}+\left[1-C-(1-C)^{(\eta+1)}\right] \rho_{s i} \\
& +(1-C)^{(\eta+1)} \rho_{b i},
\end{aligned}
$$

which can be solved for $\rho_{c l}$ and $C$, using the satellite digital count value $\rho_{t i}$, and the digital count values for $\rho_{s i}$ and $\rho_{b i}$ as just discussed. This approach thus provides a means to execute mixture decomposition without the need for atmospherically corrected, calibrated data. As long as the end member digital count values are determined and applied within a region where atmospheric opacity is homogeneous, digital count values should work. As we saw from Fig. 14, the end member proportions will not be altered by a change in the end member reflectances introduced by linear transformations such as calibration changes and atmospheric differences.

\section{CONCLUSIONS}

We believe we have demonstrated that mixture decomposition techniques, using red and NIR reflectance, can provide useful estimates of fraction of sunlit canopy, fraction of sunlit background, and fraction of shadow in pure conifer stands. The empirical and theoretical considerations herein suggest that these results should generalize beyond black spruce and jack pine stands to other conifers, and perhaps even to deciduous canopies. Secondly, we find that these radiometric fractions are highly correlated to biophysical parameters of interest; specifically, shadow and sunlit background fraction to biomass density, dbh, LAI, and aboveground NPP. The linear regression statistics are shown in Table 6. The sensitivity of the biophysical variables to the various radiometric fractions show only a mild dependence on solar zenith angle for such angles $<50^{\circ}$; thus corrections for this effect, while theoretically practical, may not be necessary. We have also shown why a popular vegetation index, NDVI, does not provide reliable estimates of stand biophysical characteristics directly; NDVI is shown to be highly correlated to the fraction of sunlit canopy, which itself is relatively insensitive to both biomass and LAI. However, because NDVI is correlated to sunlit canopy fraction, we would expect it to be a reliable measure of the fraction of photosynthetically active radiation incident upon the canopy, and thus the fraction of photosynthetically active radiation absorbed by the canopy. Finally, we have explored the issues involved in using mixture decomposition operationally in a regional or global context to estimate surface biophysical characteristics. We have investigated the effects of atmosphere on the mixture decomposition approach and have shown that when end member samples for background and shadow can be directly identified in the satellite data, no corrections for atmospheric interference are necessary to decompose the image pixels into their end member fractions; however, if absolute values for end member reflectances are used, the satellite data must be accurately calibrated and atmospherically corrected. To use mixture decomposition and geometric canopy models to estimate biophysical characteristics on a regional or global basis, however, the land surface must first be stratified into regions of homogeneous ground cover with respect to background and crown reflectance as well as crown geometry; the mixture decomposition algorithm must then be applied to each stratum, using stratum-unique values for crown and background reflectance. In addition, the relationship between the end member fractions and biophysical characteristics will depend on crown geometry. This will require the use of image processing to develop land cover maps prior to application of mixture decomposition algorithms.

\section{Literature Cited}

Allen, W. A., and A. J. Richardson. 1968. Interaction of light with a plant canopy. Journal Applied Optical Society of America 58:372-376.

Asrar, G., M. Fuchs, E. T. Kanemasu, and J. L. Hatfield. 1984. Estimating absorbed photosynthetic radiation and leaf area index from spectral reflectance in wheat. Agronomy Journal 76:300-306.

Bolle, H. J. 1991. Land surface transformation processes. Pages 181-192 in Report of the earth observation user consultation meeting, Volume SP 1143. European Space Agency, Enschede, The Netherlands.

Gates, D. M., H. J. Keegan, J. C. Schleter, and V. R. Weidner. 1965. Spectral properties of plants. Applied Optics 4:1120.

Gausman, H. W. 1984. Evaluation of factors causing reflectance differences between sun and shade leaves. Remote Sensing of Environment 15:177-181.

Gausman, H. W., and J. H. Allen. 1973. Optical parameters of leaves of 30 plant species. Plant Physiology 52:57-62.

Goel, N. S. 1988. Models of vegetation canopy reflectance and their use in estimation of biophysical parameters from reflectance data. Remote Sensing Reviews 4:1-121.

Goel, N. S., and T. Grier. 1988. Estimation of canopy parameters for inhomogeneous vegetation canopies from reflectance data: III. TRIM: a model for radiative transfer in heterogeneous three-dimensional canopies. Remote Sensing of Environment 25:255-293.

Goel, N. S., L. B. Knox, and J. M. Norman. 1990. From artificial life to real life: computer simulation of plant growth. International Journal of General Systems 18:291319 .

Goel, N. S., and R. L. Thompson. 1984. Inversion of vegetation canopy reflectance models for estimating agronomic variables. V: estimation of LAI and average leaf angle using measured canopy reflectances. Remote Sensing of Environment 16:69-85.

Goward, S. N., and K. F. Huemmrich. 1992. Vegetation canopy PAR absorptance and the normalized difference vegetation index: an assessment using the SAIL model. Remote Sensing of Environment 39:110-140.

Hall, F. G. 1994. Remote sensing and regional forest applications - a current assessment. In V. Alaric Sample, editor. 
Forest ecosystem management: the role of remote sensing and GIS. Island Press, Washington, D.C., USA.

Hall, F. G., K. F. Huemmrich, D. E. Strebel, S. J. Goetz, J. E. Nickeson, and K. D. Woods. 1992. Biophysical, morphological, canopy optical property, and productivity data from the Superior National Forest, National Aeronautics and Space Administration Technical Memorandum 104568, National Aeronautics and Space Administration Goddard Space Flight Center, Greenbelt, Maryland, USA.

Horowitz, H. M., R. F. Nalepka, P. D. Hyde, and J. P. Morgenstern. 1971. Estimating the proportions of unresolved objects within a single resolution element of a multispectral scanner 1971. Pages 1307-1320 in Proceedings of the Seventh International Symposium on Remote Sensing of Environment. Environmental Research Institute of Michigan, Ann Arbor, Michigan, USA.

Huete, A. R., R. D. Jackson, and D. F. Post. 1985. Spectral response of a plant canopy with different soil backgrounds. Remote Sensing of Environment 17:37-55.

Jasinski, M. F. 1990. Functional relation among subpixel canopy cover, ground shadow, and illuminated ground at large sampling scales. In Remote Sensing of the Biosphere. Society of Photo Optical Instrumentation Engineers, Bellingham, Washington, USA.

Jasinski, M. F., and P. S. Eagleson. 1989. The structure of red-infrared scatter grams of semi-vegetated landscapes. Institute of Electrical and Electronic Engineering Transactions Geoscience Remote Sensing 27(4):441-451.

Jasinski, M. F., and P. S. Eagleson. 1990. Estimation of subpixel vegetation cover using red-infrared scatter grams. IEEE Transactions Geoscience Remote Sensing 28(2):253267.

Kauth, R. L., and G. S. Thomas. 1976. The tasseled capa graphic description of the spectral temporal development of agricultural crops as seen by Landsat. Proceedings of the third symposium of machine processing of remote sensing data. Laboratory for Applications of Remote Sensing Purdue, 4B/41-4B/51.

Li, X., and A. H. Strahler. 1985. Geometrical-optical modeling for a conifer forest canopy. IEEE Transactions Geoscience Remote Sensing, GE-23, 705-721.

Rasool, S. I. 1992. Requirements for terrestrial biospheric data for IGBP core projects, International GeophysicalBiophysical Program-Data Information System, Paris, Working Paper 2.

Rosema, A., W. Verhoef, H. Noorbergen, and J. J. Borgesius. 1992. A new forest light interaction model in support of forest monitoring. Remote Sensing of Environment 42:2341.

Sellers, P. J. 1985. Canopy reflectance, photosynthesis, and transpiration. International Journal of Remote Sensing 6: 1335-1371.
Sellers, P., and D. Schimel. 1993. Remote sensing of the land biosphere and biogeochemistry in the EOS era: science priorities, methods and implementation-EOS land biosphere and biogeochemical cycles panels. Global and Planetary Change 7:279-297.

Shimabukuro, Y. E. 1987. Shade images derived from linear mixing models of multispectral measurements of forested areas. Dissertation. Forest and Wood Sciences, Colorado State University, Fort Collins, Colorado, USA.

Skole, D. 1992. Scientific requirements for a $1 \mathrm{~km}$ data set. Pages 11-23 in J. R. G. Townshend, editor. Improved global data for land applications. IGBP, Stockholm, Sweden.

Smith, M. O., S. L. Ustin, J. B. Adams, and R. A. Gillespie. $1990 \mathrm{a}$. Vegetation in deserts: I. a regional measure of abundance from multispectral images. Remote Sensing of Environment 31:1-26.

Smith, M. O., S. L. Ustin, J. B. Adams, and R. A. Gillespie. $1990 b$. Vegetation in deserts: II. environmental influences in regional abundance. Remote Sensing of Environment 31: $1-26$.

Suits, G. H. 1972. The calculation of the directional reflectance of a vegetative canopy. Remote Sensing of Environment 22:117-125.

Tucker, C. J., C. L. Vanpraet, E. Boerwinkle, and A. Easton. 1983. Satellite remote sensing of total dry matter accumulation in the Senegalese Sahel. Remote Sensing of Environment 13:461-469.

Ustin, S. L., M. O. Smith, and J. B. Adams. 1993.. Remote sensing of ecological processes: a strategy for developing and testing ecological models using spectral mixture analysis. Pages 339-357 in J. Ehleringer and C. B. Fields, editors. Scaling physiological processes: leaf to globe. Academic, New York, New York, USA.

Verhoef, W. 1984. Light scattering by leaf layers with application to canopy reflectance modeling: the SAIL model. Remote Sensing of Environment 16:125-141.

Wessman, C. A., J. D. Aber, D. L. Peterson, and J. M. Melillo. 1988a. Foliar analysis using near infrared reflectance spectroscopy. Canadian Journal of Forest Research 18:6-11.

Wessman, C. A., J. D. Aber, D. L. Peterson, and J. M. Melillo. $1988 b$. Remote sensing of canopy chemistry in nitrogen cycling in temperate forest ecosystems. Nature 335(6186): 154-156.

Woods, K. D., D. B. Botkin, and A. H. Fieveson. 1985. Dimension analysis: new developments in models and statistical treatments. Bulletin of the Ecological Society of America 66:297.

Woods, K. D., A. H. Feiveson, and D. B. Botkin. 1991. Statistical error analysis for biomass density and leaf area index estimation. Canadian Journal of Forest Research 21: 974-989.
Eqs. 1 and 4 can be rewritten as

$$
\begin{array}{ll}
\rho_{t 1} & =C \rho_{t 1}+S \rho_{s 1}+(1-S-C) \rho_{b 1} \quad \text { and } \\
\rho_{t 2} & =C \rho_{t 2}+S \rho_{s 2}+(1-S-C) \rho_{b 2} ;
\end{array}
$$

which can be rewritten as

$$
\begin{aligned}
& \rho_{t 1}=C\left(\rho_{t 1}-\rho_{b 1}\right)+S\left(\rho_{s 1}-\rho_{b 1}\right)+\rho_{b 1} \\
& \rho_{t 2}=C\left(\rho_{t 2}-\rho_{b 2}\right)+S\left(\rho_{s 2}-\rho_{b 2}\right)+\rho_{b 2} .
\end{aligned}
$$

Now, for $C=$ a constant, Eqs. 2 are of the form:

\section{APPENDIX}

$$
\begin{aligned}
& \rho_{t 1}=a_{1}+e_{1} S \quad \text { and } \\
& \rho_{t 2}=a_{2}+e_{2} S
\end{aligned}
$$

which can be solved for $\rho_{t 2}$ in terms of $\rho_{t 1}$ :

$$
\rho_{t 2}=d_{21}+a_{1} / e_{1} \rho_{t 1}
$$

which is, of course, the equation for a straight line with slope $a_{1} / e_{1}$ and intercept, where:

$$
\begin{aligned}
d_{21}= & C\left(\rho_{t 2}-\rho_{b 2}\right)-\left\{C\left(\rho_{t 1}-\rho_{b 1}\right)+\rho_{b 1}\right\} \\
& \cdot\left\{\left(\rho_{s 2}-\rho_{b 2}\right) /\left(\rho_{s 1}-\rho_{b 1}\right)+\left(\rho_{s 2}-\rho_{b 2}\right) /\left(\rho_{s 1}-\rho_{b 1}\right) \rho_{t 1}\right\}
\end{aligned}
$$

Similar proofs hold for $S=$ a constant and $B=$ a constant. 